\title{
M2 Macrophagy-derived Exosomal MiRNA-5106 Induces Bone Mesenchymal Stem \\ Cells towards Osteoblastic Fate by Targeting Salt- inducible Kinase 2 and 3
}

\author{
Yuan Xiong \\ Wuhan Union Hospital \\ Lang Chen \\ Wuhan Union Hospital \\ Chenchen Yan \\ Wuhan Union Hospital \\ Wu Zhou \\ Wuhan Union Hospital
}

Tao Yu

Tongji University School of Medicine

Yun Sun

Wuhan Union Hospital

Faqi Cao

Wuhan Union Hospital

Hang Xue

Wuhan Union Hospital

\section{Yiqiang Hu}

Wuhan Union Hospital

\section{Dong Chen}

Wuhan Union Hospital

Bobin Mi ( $\sim$ mibobin@hust.edu.cn )

Wuhan Union Hospital

Guohui Liu ( $\square$ liuguohui@hust.edu.cn )

Wuhan Union Hospital https://orcid.org/0000-0002-2013-1396

\section{Research}

Keywords: Exosome, MiR-5106, Osteoblast, Fracture, SIK2, SIK3

Posted Date: March 24th, 2020 
DOI: https://doi.org/10.21203/rs.3.rs-18281/v1

License: (c) (1) This work is licensed under a Creative Commons Attribution 4.0 International License. Read Full License

Version of Record: A version of this preprint was published at Journal of Nanobiotechnology on April 28th, 2020. See the published version at https://doi.org/10.1186/s12951-020-00622-5. 


\section{Abstract}

Background Osteoblast differentiation is a vital process for fracture healing, and exosomes are nanosized membrane vesicles that can deliver therapeutic drugs easily and safely. Macrophages participate in the regulation of various biological processes in vivo, and macrophage-derived exosomes (MD-Exos) have recently been a topic of increasing research interest. However, few study has explored the link between MD-Exos and osteoblast differentiation. Herein, we sought to identify miRNAs differentially expressed between M1 and M2 macrophage-derived exosomes, and to evaluate their roles in the context of osteoblast differentiation.

Results We found that microRNA-5106 (miR-5106) was significantly overexpressed in M2 macrophagederived exosomes (M2D-Exos), while its expression was decreased in M1 macrophage-derived exosomes (M1D-Exos), and we found that this exosomal miRNA can induce bone mesenchymal stem cell (BMSC) osteogenic differentiation via directly targeting the Salt-inducible kinase 2 and 3 ( SIK2 and SIK3) genes. In addition, the local injection of both a miR-5106 agonist or M2D-Exos to fracture sites was sufficient to accelerate healing in vivo.

Conclusions Our study demonstrates that miR-5106 is highly enriched in M2D-Exos, and that it can be transferred to BMSCs wherein it targets SIK2 and SIK3 genes to promote osteoblast differentiation.

\section{Background}

Fracture is very common in clinic, which can bring heavy burden to fracture patients. The rapid healing of fracture can help patients to get early recovery, relieve the financial burden, and improve the quality of life. Bone mesenchymal stem cells (BMSCs) are capable of differentiating into osteoblasts, making them crucial in the process of fracture healing. $[1,2]$ Previous studies have found that many factors contribute to the differentiation of BMSCs, including microRNA-188, modulates age-related switching between osteoblast and adipocyte differentiation. [3] Similarly, myocardin-related transcription factor A (MRTFA) has been found to be a positive regulator of BMSCs. [4] The identification of further factors regulating BMSCs differentiation may therefore allow for a better understanding of osteoblast differentiation. Macrophages are vital for all stages of fracture healing. [5] They are among the earliest cells to migrate to fracture sites, stimulated by the tissue injury and the associated acute inflammatory phase. [6] Macrophages are also necessary for osteoblast differentiation in vitro, and have been shown to be important during both early and late stages of fracture healing. [7, 8] Particularly important for the healing process is macrophage phenotypic switching from the more inflammatory M1 subtype to the antiinflammatory $\mathrm{M} 2$ subtype, with non-union often being associated with prolonged pro-inflammatory macrophage responses. $[9,10]$ However, the mechanisms governing the beneficial role of $M 2$ macrophages in the context of osteoblasts differentiation remain elusive.

Nanomaterials, which could exert certain regulatory function in vivo, had obtained accumulated evidence of their roles in osteogenic differentiation. [11-13] Exosomes represent a promising type of 
nanomaterials from which miRNAs can be isolated. [14] MiRNAs in turn are able to bind with target mRNAs to regulate their rates of expression or degradation without being interfered by various of internal and external factors. [15] Indeed, miRNAs have been reported to be secreted extracellularly in exosomes and to exert a regulatory role in a wide array of biological processes. $[16,17]$ One recent study, for example, found that myocardial miRNAs encapsulated in circulating exosomes coordinate systemic responses to cardiac injuries, potentially offering novel therapeutic avenues for treating myocardial infarction. [18]

Herein, we sought to investigate the effect of M2 macrophage-derived exosomal miRNAs on BMSCs differentiation. We demonstrate that miR-5106 is highly enriched in M2D-Exos, and that it can be transferred to BMSCs wherein it targets the SIK2 and SIK3 genes to induce osteoblastic differentiation in vitro and in vivo.

\section{Results}

\section{M1 and M2 macrophages secrete exosomal miRNAs}

We first assessed the expression of phenotypic markers associated with bone marrow-derived macrophages (BMDMs) by flow cytometry (Supplementary Figure 1A). To monitor M1 polarization, we assessed the expression of phenotypic markers associated with M1 macrophages, including F4/80 and $\mathrm{CD} 11 \mathrm{c}$. As expected, the rate of $\mathrm{F} 4 / 80$ and $C D 11 \mathrm{c}$ dual-positivity in BMDMs was significantly increased after 24 hours of treatment with LPS and IFN- $y$ (Supplementary Figure 1B). Similarly, the rate of F4/80 and CD206 dual-positivity in BMDMs was significantly increased after 24 hours of treatment with IL-4 (Supplementary Figure 1C). These results confirmed the success of the cellular M1 and M2 polarization model we used.

We next assessed the ability of these macrophages to secrete miRNAs that can be internalized by other cells. To do this, we used a model system wherein miRNA-26a-5p (miR-26a-5p), which is an osteoblastselective miRNA, ${ }^{19}$ was labeled with Cy3 and transfected into these M1 and M2 macrophages in the upper chamber of a transwell chamber system. BMSCs were added to the lower chamber, and we assessed the delivery of Cy3-miR-26a-5p from the upper to the lower chamber in this assay system. As we observed increasing red fluorescence among BMSCs over time, which confirmed that miR-26a-5p was released from both types of macrophages in a format that was internalized by BMSCs (Figure 1A). In contrast, when free Cy3 was used to treat M1 or M2 macrophages prior to their use in this assay system, minimal Cy3 was detectable in BMSC2 following a 12 hours co-culture (Supplementary Figure 2). As such, these results show that M1 and M2 macrophages can secrete extracellular miRNAs that can be internalized by BMSCs.

To explore whether M1 and M2 macrophage secrete exosomes, we extracted exosomes via ultracentrifugation and analyzed the isolates via transmission electron microscopy (TEM), dynamic light scattering (DLS), and flow cytometry. DLS suggested particles with sizes ranging from 30-200 nm were 
present within samples (Figure 1B). TEM revealed these particles to have cup- or sphere-shaped morphology (Figure 1C). Flow cytometry analysis further give evidence that exosomal surface markers such as CD63 and CD81 were present on these particles (Figure 1D). Together these results suggested that the isolated circulating nanoparticles are exosomes.

Subsequently, we tested whether these MD-Exos can be taken up by BMSCs. These MD-Exos were labeled with the fluorescent dye PKH26 and then added into the culture medium of BMSCs. After 12 hours, the BMSCs exhibited efficient uptake of the MD-Exos, as indicated by the presence of red fluorescence staining in these cells (Figure 1E). Together these results suggested that macrophages can secrete miRNA-containing exosomes, which are then efficiently transported into recipient cells.

\section{M1D-Exos and M2D-Exos regulate BMSCs differentiation}

We next investigated how MD-Exos affect osteogenic differentiation in vitro. BMSCs were treated with phosphate buffered saline (PBS), $100 \mu \mathrm{g} / \mathrm{ml}$ M1D-Exos, or $100 \mu \mathrm{g} / \mathrm{ml}$ M2D-Exos respectively. Expression of the osteogenic genes collagen I, alkaline phosphatase (ALP), osteocalcin (OCN), and runt-related transcription factor 2 (Runx2) were then measured by western blotting and quantitative reverse transcriptase PCR (qRT-PCR) 48 hours post transfection. As compared with the PBS group, significantly higher mRNA levels of these genes were detectable in the M2D-Exos group, while levels were lower in the M1D-Exos group (Figure 2A to 2C). To explore the impact of M1D-Exos and M2D-Exos on extracellular matrix mineralization, cells were continuously cultured for 21 days and then assessed for alizarin red staining, revealing reduced mineral deposition in M1D-Exos-treated cells, and enhanced mineral deposition in M2D-Exos-treated cells (Figure 2D and 2F). Similarly, M1D-Exos reduced ALP activity and staining, whereas M2D-Exos enhanced ALP activity and staining (Figure 2E and 2G).

\section{M2D-Exos accelerates murine femoral fracture healing}

To explore the impact of M1D-Exos and M2D-Exos on murine fracture healing, fracture mice model were locally injected a total of $100 \mu \mathrm{l}$ samples with PBS, $100 \mu \mathrm{g} / \mathrm{ml}$ M1D-Exos, or $100 \mu \mathrm{g} / \mathrm{ml} \mathrm{M2D-Exos}$ respectively in the fracture sites on days 0,4 , and 7 after surgery. X-rays and micro-CT examinations were used to monitor the process of fracture healing. Mice treated with M2D-Exos exhibited a larger callus volume and smaller fracture gap compared with control animals (Figure 3A and 3B). Furthermore, on day 21, bone samples were collected for qRT-PCR analysis of osteogenic genes, revealing that levels of collagen I, ALP, OCN, and Runx2 were increased significantly in M2D-Exos-treated animals relative to those in the other treatment groups (Figure $3 \mathrm{C}$ ). In addition, bone samples were further collected for Hematoxylin and Eosin (H\&E)/Alcian-blue staining on days 21post-surgery. H\&E/Alcian-blue staining results revealed a reduced cartilage area and increased bone area at the fracture junction in the M2D-Exo group, suggesting more rapid remodeling for these animals (Figure 3D and 3E). Moreover, M2D-Exotreated animals exhibited significantly higher bone volume (BV), total volume (TV), BV/TV, and bone mineral density (BMD) relative to other study animals (Figure 3F). Together these results suggested that M2D-Exos mediated accelerated fracture healing. 


\section{Microarray-mediated identification of miRNAs differentially expressed in M1D- and M2D-Exos}

To identify miRNAs differentially expressed between M1D-Exos and M2D-Exos, we first extracted M1DExos and M2D-Exos via ultracentrifugation method, and then used a miRNA microarray approach to compare the miRNA expression profiles therein. Unsupervised clustering of the microarray results revealed significantly distinct miRNA profiles between M2D-Exos and M1D-Exos (Figure 4A and 4B). We then identified miRNAs with a mean fold change $>5$ or $<-5$ and a $p$ value $<0.05$ for further analyses. Based on the criteria, miR-709, miR-3112-3p, miR-3069-3p, miR-6406, miR-6389, miR-5106, miR-8103, miR-1934-3p, miR-22-3p, and miR-505-5p were the top ten overexpressed miRNAs. The expression of these top 10 miRNAs was then validated via qRT-PCR in six independent M1D-Exo and M2D-Exo samples, revealing miR-5106 to be significantly upregulated in M2D-Exos relative to M1D-Exos, leading us to study it further (Figure 4C). We compared miR-5106 expression between M1 and M2 macrophage via qRT-PCR analysis, confirming its overexpression in the M2 macrophage group (Figure 4D). Furthermore, we cocultured M1 or M2 macrophages with BMSCs in a transwell system as above, and a significant increase in miR-5106 abundance in the BMSCs was detectable following M2 macrophage co-culture (Figure 4E). In addition, BMSCs were divided into four groups and subjected to four different treatments (PBS only, M2DExos, antagomiR-5106, antagomiR-5106+M2D-Exos), after which miR-5106 expression was assessed via qRT-PCR analysis, revealing that M2D-Exos were able to partially reverse the antagomiR-5106-mediated reduction in miR-5106 levels in these cells (Figure 4F). As demonstrated above, MD-Exos can be internalized by BMSCs. Thus, these results suggested that miR-5106 is enriched in M2D-Exos and can be delivered to BMSCs by containing in M2D-Exos.

\section{MiR-5106 induces BMSCs differentiation in vitro}

Next, we explored the ability of miR-5106 to directly affect BMSCs by treating cells with PBS, control agomiR construct (agomiR-NC), agomiR-5106, control antagomiR construct (antagomiR-NC), or antagomiR-5106 respectively, and the results indicated that the agomiR-5106 treatment significantly increasing the miR-5106 expression in BMSCs (Figure 5A). Osteogenic related genes, including collagen I, ALP, OCN, and Runx2, were then measured by western blotting and qRT-PCR analysis, revealing a clear increase in these genes in cells in the agomiR-5106 group (Figure 5B to 5D). Moreover, we treated M2 macrophages with miR-5106 inhibitor (antagomiR-5106) to suppress the expression of miR-5106 in the cells, and then extract the exosomes from these cells to investigate the effect of M2D-Exos with miR5106-silence on the osteoblastic differentiation of BMSCs. The results showed that antagomiR-5106 can significantly decrease the miR-5106 expression in M2D-Exos, and decreased osteogenic genes expression can be detected in M2D-Exos ${ }^{\text {antagomiR-5106 }}$ group compared with PBS and M2D-Exos groups (Figure 5E and 5F). Additionally, to explore the roles of miR-5106 on extracellular matrix mineralization, cells were continuously cultured for 21 days and then analyzed via alizarin red staining, revealing enhanced mineral deposition in agomiR-5106-treated cells (Figure 5G). Similarly, miR-5106 induced more pronounced ALP activity and staining (Figure $5 \mathrm{H}$ ). Together these results thus confirmed the ability of miR-5106 to directly induce BMSCs differentiation in vitro. 
We further assessed the molecular mechanisms whereby miR-5106 promotes BMSCs functionality. To do so, TargetScan (http://www.targetscan.org/vert_70/) was used to identify putative miR-5106 targets. Following a literature review, we found SIK2 and SIK3 to be the most associated with osteogenic differentiation among these candidate genes. [20] Furthermore, a luciferase reporter assay was used to confirm that miR-5106 was able to specifically bind to the predicted target region of the SIK2 and SIK3 mRNAs such that when these regions were mutated, the miRNAs were no longer able to bind to suppress luciferase activity (Figure 6A). Moreover, we found that miR-5106 overexpression led to a clear suppression of SIK2 and SIK3 expressions (Figure 6B and 6C, Supplementary Figure 3A). To explore the dependence of osteoblastogenesis on SIK2 and SIK3 genes, we used a SIK2-specific and SIK3-specific siRNA to examine its role in this process (Figure 6D and 6E, Supplementary Figure 3B). SIK2 and SIK3 knockdown resulted in elevated collagen I, ALP, OCN, and Runx2 expression (Figure 6F and 6G, Supplementary 3C). Moreover, SIK2 and SIK3 expressions in mice callus decreased significantly after receiving the injection of agomiR-5106 compared with PBS and antagomiR-5106 groups (Figure $6 \mathrm{H}$ and 6I, Supplementary 3D). However, when over-expression of SIK2/SIK3, decreased levels of osterogenic genes can be detected in BMSCs, and their negative effects on osteogenic differentiation can be partly rescued by agomiR-5106 (Figure 6J to $6 \mathrm{~L}$ ). In addition, we further explored the impact of SIK2 and S/K3 genes on extracellular matrix mineralization. After growth in for 21 days in osteoblast inducing conditional media, stronger mineral deposition was evident for cells treated with SIK2-specific siRNA and SIK3-specific siRNA relative to controls (Figure 6M). Similarly, SIK2-specific siRNA and SIK3-specific enhanced ALP activity and staining (Figure 6N).

\section{M2D-exos-derived miR-5106 accelerates fracture healing in vivo}

To investigate how M2D-Exos-derived miR-5106 affects fracture healing, we administered PBS, M2D-

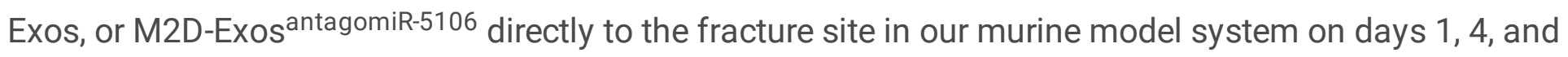
7 post-injury. Animals were then monitored via X-rays and micro-CT approaches, we observed a significantly reduced fracture gap and a larger callus volume in mice treated using M2D-Exos, but not M2D-Exos ${ }^{\text {antagomiR-5106 }}$, compared with control group. And on day 21 post-fracture, animals treated using M2D-Exos no longer exhibited a clear boundary between hardened callus and cortical bone, and remodeling had clearly taken place. However, when silencing the expression of miR-5106 in M2D-Exos, the positive effect was mainly reversed (Figure 7A and 7B). Furthermore, on day 14, bone samples were collected for qRT-PCR analysis of miR-5106, revealing that levels of miR-5106 was increased significantly in M2D-Exos-treated animals relative to those in the other treatment groups (Figure 7C). In addition, H\&E/Alcian-blue staining on days 21 further indicated reduced cartilage area at the fracture site for mice treated with M2D-Exos group and knocked out miR-5106 can impair this po-fracture-healing effect, consistent with this miRNA facilitating more rapid remodeling (Figure 7D and 7E). Moreover, on day 14 in M2D-Exos antagomiR-5106-treated animals the total and bone callus volume was smaller relative to mice in M2D-Exos groups, and this remained significant when comparing M2D-Exos ${ }^{\text {antagomiR-5106 }}$ and M2D-Exos 
mice on day 21 post-injury (Figure 7F). BMD was also significantly higher for mice treated with M2D-Exos relative to animals in other groups (Figure 7F). This suggested that miR-5106 derived from M2D-Exos positively regulates fracture healing.

\section{Discussion}

Macrophage is a cell type that can readily undergo differentiation into a range of effector subtypes depending on local cellular and secreted signals. The positive regulatory role of $\mathrm{M} 2$ macrophages during bone fracture healing is already well known. [19-21] However, the underlying mechanisms for this beneficial role remain elusive. To more fully explore the role of $M 2$ macrophages in bone formation and to explore the potential underlying mechanisms, we herein focused on the effects of M2D-Exos on osteoblast differentiation in vitro and in vivo. Exosomes are small vesicles that are secreted into circulation by a range of cell types in vivo, whereupon they can be internalized by proximal or distal cells. [22] The small molecules within these exosomes (including proteins and nucleic acids) can then regulate the functionality of recipient cells upon internalization, and thereby conducting a communication among various cells and organs. [23] In addition, exosomes have been demonstrated to be one of the ideal nanomaterials for delivering the regulatory substances to the targets and acted as a protective role in this process. [24] Our results suggested that M2D-Exos were able to stimulate osteoblast differentiation and bone mineral deposition, as well as to promote fracture healing in murine models. All these data thus suggest that the ability of M2D-Exos to induce osteoblast function may explain the previously reported benefits of M2 macrophages in the context of fracture healing, as well as providing a novel therapeutic strategy for the treatment of fracture.

miRNAs are small RNA molecules that cannot encode protein but play vital roles in diseases such as cancer and processes such as immunoregulation and inflammation. [24-26] In immune system, miRNA expression patterns can be used as a biomarker for particular disease states such as cancer or endocrine events. [27, 28] Evidence also reported that exosomes can be taken up into neighboring or distant cell types to modulate the function of recipient cells. [29] Therefore, to explore the relationship between macrophage-derived exosomal miRNAs and BMSCs, we co-cultured these two cell types, revealing that exosomal miRNAs from macrophages could be internalized by BMSCs, providing a strong basis for our further research. In the present research, we searched out miR-5106 to be the key miRNA enriched in M2DExos by using microarray analysis. There is few research had reported the functionality of miR-5106 previously. To get the knowledge of this miRNA, we screened the NCBI database (https://www.ncbi.nlm.nih.gov/gene/100628618). It reported that miR-5106 is involved in posttranscriptional regulation of gene expression in multicellular organisms by affecting both the stability and translation of mRNAs. Therefore, we explored the target mRNA of miR-5106 by using TargetScan system, and the genes SIK2 and SIK3 were selected to be the potential target genes for miR-5106. SIK2 and SIK3 were demonstrated involved in various processes such as cell cycle regulation, gluconeogenesis and lipogenesis regulation, muscle growth and differentiation and tumor suppression. [30, 31] And a recent research had indicated that SIK2 and SIK3 inhibition is associated with the enhance of antiinflammatory phenotype of macrophages. [31] In the present study, we demonstrated the relationship of 
M2D-Exos-derived miR-5106 and SIK2 and SIK3 genes by using luciferase assays, and the negative regulatory effect of miR-5106 on SIK2 and SIK3 expression was then verified in vitro.

Osteoblast differentiation is a vital process for fracture healing, arising as a result of various intersecting factors. [32] We observed the upregulation of miR-5106 in exosomes isolated from M2 macrophages, and we found that this miRNA positively regulated osteogenic differentiation. When miR-5106 was transferred into BMSCs, their ability to undergo such differentiation was markedly enhanced. Similarly, we further observed that miR-5106 was able to accelerate fracture healing in vivo. Several regulators have been reported to mediate the osteogenic differentiation of BMSCs, [33, 34] with SIK2 and SIK3 genes being the two of the most important such factors. $[35,36]$ Herein, we found that SIK2 and SIK3 were the miR-5106 target genes, thus highlighting a mechanistic role for miR-5106 in mediating osteoblastic differentiation. When downregulated, SIK2 and SIK3 are no longer able to suppress the osteoblastic differentiation of BMSCs, and thereby accelerating fracture healing in vivo.

\section{Conclusions}

Taken together, our results indicate that exosomes isolated from the M2 macrophages are capable of accelarating fracture healing via interference with BMSC differentiation in vitro and in vivo. This positive effect is at least partially mediated by miR-5106, which is enriched in M2D-Exos and can promote osteogenic differentiation of BMSC via suppressing the expression of SIK2 and SIK3 (Fig. 8). Therefore, our findings suggest that local injection of M2D-Exos may represent a promising therapeutic strategy to bolster fracture healing.

\section{Methods}

\section{Culture of macrophages}

The Institutional Review Board at Union Hospital, Tongji Medical College, Huazhong University of Science and Technology approved this study. C57BL/6J mice (6-week-old) from the Center of Experimental Animals, Tongji Medical College, Huazhong University of Science and Technology were used for this study. BMDMs were generated using M-CSF as in past reports. ${ }^{[26]}$ Briefly, bone marrow cells isolated from these mice were grown in RPMI-1640 (\#11875093, Gibco, Grand Island, USA) containing 10\% FBS (\#10099141, Gibco, Grand Island, USA), 1\% penicillin/streptomycin (\#SV30010, Hyclone, USA), and 50 ng/ml M-CSF (\#216-MC, R\&D System, Minneapolis, MN, USA) for 7 days. Subsequently, BMDMs were harvested for flow cytometric and transcriptomic analysis. M1 and M2 macrophages differentiation was initiated by treating BMDMs for 24 hours using either 100ng/ml LPS (\#L2630, Sigma-Aldrich, St Louis, MO, USA) + 20ng/ml IFNY (\#285-IF-100/CF, R\&D Systems), or 20ng/ml IL-4 (\#204-IL-010, R\&D Systems), respectively. M1 and M2 macrophages were then harvested for flow cytometric and transcriptomic analysis.

\section{Flow Cytometric Analysis}


This procedure was performed as descripted previously.[27] Antibodies were purchased from eBioscience company. BMDMs, M1 macrophages, and M2 macrophages were stained using lineage-specific antibodies, with F4/80 (\#123107, Biolegend, San Diego, CA, USA) and CD 209 (\#12-2092-80, SigmaAldrich) used for BMDM identification, F4/80 and CD11C (\#117307, Biolegend) used for M1 macrophage identification, and F4/80 and CD206 (\#141705, Biolegend) used for M2 macrophage identification. FlowJo software (version: FlowJo ${ }^{\mathrm{TM}} \mathrm{v}$ 10.6.1) was used for data analysis.

\section{BMSCs Culture and Transfection}

BMSCs were kindly donated by the Huazhong University of Science and Technology, Wuhan, China. Cells were grown in a specific media designed for C57BL/6 mouse mesenchymal stem cells (\#MUBMX-03011440 , Cyagen, Guangzhou, China) at $37^{\circ} \mathrm{C}$ in a $5 \% \mathrm{CO} 2$ incubator. Cells were maintained for a maximum of 3 passages. Lipofectamine 3000 (\#L3000001, ThermoFisher Scientific, USA) was used to transfect cells with siRNAs or miRNAs based on provided directions. agomiR-5106, agomiR-NC, antagomiR-5106, and antagomiR-NC (GenePharma, Shanghai, China) were transfected into cells using a $20 \mathrm{uM}$ final concentration, whereas SIK2 siRNA, and SIK3 siRNA constructes (RIBOBIO, Guangzhou, China) were transfected at $50 \mathrm{nM}$. Plasmid-NC, plasmid-SIK2, and plasmid-SIK3 were synthesized by GenePharma (GenePharma, Shanghai, China).

\section{Exosome purification, characterization and uptake}

Following a 72 hours culture step, we removed debris and dead cells from samples via a 10 minutes spin at $1000 \mathrm{xg}$, followed by filtration with a $0.2 \mu \mathrm{m}$ filter (122-0020PK ThermoFisher Scientific). Media then underwent ultracentrifugation for 6 hours at $100,000 \times g$ at $4^{\circ} \mathrm{C}$. Samples were then washed with PBS and spun for an additional 20 minutes at 100,000 xg, after which PBS was used to resuspend the pellet, which contained exosomes. Transmission electron microscopy (TEM; EFI, TECNAI G2) was used to assess morphology, while a Nanosizer ${ }^{\text {TM }}$ instrument (Malvern Instruments, Malvern, UK) was used for Dynamic light scattering (DLS) analyses and flow cytometry was used to assess exosome surface marker expression. BMSC uptake of these exosomes was confirmed by labeling isolated exosomes using PKH 26 (\#MINI26-1KT, Sigma, MO, USA) and then collecting them via centrifugation at 110,000 $\mathrm{xg}$ for 20 minutes. Briefly, exosomes were suspended in $1 \mathrm{~mL}$ diluent $\mathrm{C}$ containing $5 \mu \mathrm{M} \mathrm{PKH} 26$ and incubated for 5 minutes. The labeling action was stopped after 1 minute incubation with an equal volume of $1 \%$ bovine serum albumin (Bovogen, Melbourne, Australia). The exosomes were washed three times with Amicon ultrafilter (10 KDa cut-off, Millipore, MA, USA) with cold PBS and then resuspended in $200 \mu \mathrm{L}$ PBS. These labeled exosomes were used to treat BMSCs for 12 hours followed by analysis under a confocal microscope.

\section{qRT-PCR analysis}

Before extracting miRNAs, callus samples were preserved using RNA Later (\#76104, QIAGEN, Germany). Trizol (\#15596018, Invitrogen, USA) was used to isolate total cell RNA, after which a Verso ${ }^{\text {TM }}$ CDNA Synthesis Kit (\#AB-1054/A, ThermoFisher Scientific) was applied to reverse transcribe RNA. miRNA 
expression was assessed using the SeraMir Exosome RNA purification Kit (System Biosciences, USA) to extract exosomal miRNAs, followed by use of a TaqMan microRNA assay kit (Applied Biosystems, USA) for cDNA synthesis. All qRT-PCR reactions were performed using a Thermal Cycler C-1000 Touch system (\#10021377, Bio-Rad CFX Manager, USA), and U6 or GAPDH were used to normalize target expression as appropriate. Data were expressed as fold changes over controls. Primers used herein are compiled in Table 1.

\section{Luciferase reporter assays}

BMSCs were added to 24 -well plates $\left(2.5 \times 10^{5}\right.$ cells/well) followed by transfection with dual-luciferase vectors (SIK2 WT, SIK2 Mut, SIK3 WT, and SIK3 Mut) along with either miR-5106 agonist (AgomiR-5106) or negative control (AgomiR-NC). A Quik Change Site-Directed Mutagenesis Kit (Strata gene) was used to insert mutations in the binding-region and dual luciferase reporter assay (Promega) was performed according to the manufacturer's instructions. Ultimately, a luminometer (Glomax, Promega) was used to quantify luminescence, normalizing each value from the firefly luciferase construct to the corresponding Renilla signal.

\section{Western blotting}

Lysis buffer (\#AS1004, Aspen, South Africa) containing 1\% protease inhibitor (\#AS1008, Aspen) was used to lyse cells or callus samples, after which protein was separated via SDS-PAGE and transferred to NC membranes (\#IPVH00010, Millipore, USA) that were blocked with 5\% nonfat milk and stained overnight at $4^{\circ} \mathrm{C}$ overnight with antibodies specific for collagen I (1:500, Sigma, USA, \#ab34710), ALP (1:1000, Sigma, USA,\#ab95462), Osteocalcin (1:500, Sigma, USA, \#ab93876), RunX2 (1:500, Sigma, USA, \#ab23981), SIK2 (1:1,000, Sigma, USA, \#SAB1302059), SIK3 (1:1,000, Sigma, USA, \#SAB3500695), and GAPDH (1:10,000, Sigma, USA, \#ab37168). Blots were then stained with appropriate secondary antibodies conjugated to horseradish peroxidase (HRP) (\#AS1058, Aspen), and proteins were detected with a chemiluminescence detection system. Each experiment was repeated three times.

\section{Alizarin red staining}

BMSCs were grown in 6-well plates in media containing $100 \mathrm{nM}$ dexamethasone, $50 \mathrm{mM}$ ascorbic acid, and $10 \mathrm{mM}$ b-glycerophosphate to promote osteogenesis (\#HUXMA-90021, Cyagen, USA). Briefly, cells were washed twice by using PBS, after wich $10 \%$ formalin was added to fix the cells for 15 minutes. Subsequently, $1 \mathrm{~mL} 0.5 \%$ alizarin red staining solution was used to stain the cells at room temperature for 15 minutes. After rinsing with distilled water for 5 minutes, red mineralized nodules were analyzed via charge-coupled device microscope. Absorbance was then measured at $570 \mathrm{~nm}$. Experiments were repeated in triplicate.

\section{ALP staining}


A BCIP/NBT alkaline phosphatase color development kit (\#C3206, Beyotime, China) was utilized based upon provided directions. Briefly, cells were washed twice by using PBS, after wich $10 \%$ formalin was added to fix the cells for 15 minutes. BCIP/NBT substrate was then used to treat cells for $24 \mathrm{~h}$, and colorimetric changes were analyzed using a charge-coupled microscope, with a scanner used to image stained cells. Absorbance was then measured at $405 \mathrm{~nm}$. Experiments were repeated in triplicate.

\section{Mice fracture model and treatment}

Male C57BL/6J mice (8-week-old) were obtained as above, with all studies being approved by the Institutional Animal Care and Use Committee at Tongji Medical College, Huazhong University of Science and Technology. Intraperitoneal pentobarbital sodium (50 mg/kg; Sigma-Aldrich) was used to anesthetize animals, and a model of femoral fracture was then generated via longitudinal incision and blunt separation of the underlying muscles without periosteum removal, as in previous reports ${ }^{28}$. The femur was then cut with a diamond disk, yielding a mid-diaphysis transverse osteotomy. Next, a 23-gauge intramedullary needle was used to stabilize the fracture site. On day 14 post-fracture, $50 \%$ of study animals were euthanized to harvest and analyze calluses, with the remaining mice being analyzed on day 21.

\section{Radiographic images}

On days 7,14 , and 21 post injury, all animals were subjected to $X$ rays with an In-Vivo FX PRO imaging system (BRUKER, Karlsruhe, Germany) with a 10 second exposure time.

\section{Micro-computer tomography (Micro-CT) analysis}

A BRUKER SkyScan 1276 scanner micro-CT system (BRUKER, Karlsruhe, Germany) was used to image fracture regions in mice (2400 views, 5 frames/view, $37 \mathrm{kV}$, and $121 \mathrm{~mA}$ ), with the platform software being used to assess segmentation, three dimensional morphometric analyses, density, and distance parameters (BRUKER, Karlsruhe, Germany). After scanning, preserved calluses were stored at $-80^{\circ} \mathrm{C}$ prior to further experimental use. Measure parameters, includeding bone volume (BV), total volume (TV), BV/TV, and bone mineral density (BMD), were analyzed by CT Analyser evaluation software (Version: 1.15.4.0) on day 14 and day 21 post-operation. Micro-CT analysis was done in a completely blinded manner with all mice assigned to coded sample numbers.

\section{Therapeutic administration in fracture mice}

Control mice received a local fracture site injection of $0.1 \mathrm{~mL}$ PBS on days 1, 3, and 7 post-fracture, while other mice were instead administered equivalent volumes of M1D-Exos, M2D-Exos, M2D-Exos antagomiR5106. At the end of the experiment, bone and callus samples were analyzed as above.

\section{Histological analysis}


Following decalcification, 5-7 $\mu \mathrm{m}$ thick paraffin-embedded tissue samples were prepared and subjected to H\&E and Alcian blue staining. Sections were then imaged and measured with an Olympus BX51 microscope and a DP73 CCD Olympus Imaging System (Olympus Corporation, Tokyo).

\section{Microarray analysis}

RNA sequencing from M1 and M2 macrophages' supernatant was conducted using a BGISEQ500 platform (BGI). Downstream library was constructed by using RNA samples with RNA integrity number values > 8.0. mRNAs were isolated and reverse transcription with PCR amplification was performed. Then, cDNA was obtained as the final sequencing library, and verified on a bioanalyzer (Agilent 2100). DNA nanoballs were produced by phi29 and the library was amplified, which were loaded into the patterned nanoarray followed by SE50 sequencing. Differentially expressed miRNAs $\triangle D E M s \rrbracket$ with statistical significance between the two groups were identified by volcano plot filtering and fold change filtering was used to identify DEMs between the two samples. The Gene Cluster 3.0 software (Stanford University) was applied to make hierarchical cluster analysis. Database of annotation, visualization, and integrated discovery (DAVID 6.8; https://david.ncifcrf.gov/) was used to conduct functional analysis. The $p$ value was set to 0.05 to denote the significance of GO enrichment of DEMs.

\section{Statistical analysis}

Data are means \pm SD, and GraphPad Prism 8.0 (GraphPad Software, CA, USA) was used for all statistical testing. Data were compared via Student's t-tests and one-way ANOVAs with Tukey's post-hoc test as appropriate. $\mathrm{P}<0.05$ was the significance threshold.

\section{Declarations}

\section{Funding}

This study was supported by the National Science Foundation of China (No.81772345), National Health Commission of the People's Republic of China (No. ZX-01-018\ZX-01-C2016153), Ministry of Science and Technology of the People's Republic of China (No.2018YFC2001502, 2018YFB1105705), Health Commission of Hubei Province (No.WJ2019Z009), Wuhan Science and Technology Bureau (No.2017060201010192).

\section{Acknowledgement}

We thank Jing Liu, Abududilibaier Abudula, Ranyang Tao, Zexi He, Xingjie Zhan, and Chenming Zhang for assistance with data collection.

\section{Conflicts of interests}

All authors declared no conflict of interest.

\section{Availability of data and materials}


All data generated or analyzed during this study are included in this published article.

\section{Consent for publication}

All authors agree to be published

\section{Ethics approval and consent to participate}

Not applicable.

\section{Contributor information}

Yuan Xiong, Email: xiongyuanmed@163.com

Lang Chen, Email: langchen199508@163.com

Chenchen Yan, Email: Naruto_17@163.com

Wu Zhou, Email: wuzhoutjmu1986@163.com

Tao Yu, Email: yutao247@tongji.edu.cn

Faqi Cao, Email: 13971293030@163.com

Yun Sun, Email: 627224540@qq.com

Hang Xue, Email: 15871393021@163.com

Yiqiang Hu, Email: 472976834@qq.com

Bobin Mi, Email: mibobin@hust.edu.cn

Guohui Liu, Email: liuguohui@hust.edu.cn.

\section{References}

1. Milovanovic P, Zimmermann EA, Vom Scheidt A, Hoffman, B,Sarau G, Yorgan T, Schweizer M, Amling $M$, Christiansen $S$, Busse $B$, The formation of calcified nanospherites during micropetrosis represents a unique mineralization mechanism in aged human none, Small. 2017; 13: 02215-39.

2. Kim KI, Park S, Im GI, Osteogenic differentiation and angiogenesis with cocultured adipose-derived stromal cells and bone marrow stromal cells, B 2014; 35: 4792-4804.

3. Li CJ, Cheng P, Liang MK, Chen YS, Lu Q, Wang JY, Xia ZY, Zhou HD, Cao X, Xie H, Liao EY, Luo XH, MicroRNA-188 regulates age-related switch between osteoblast and adipocyte differentiation, $J$ Clin Invest. 2018; 125: 1509-1522. 
4. Bian H, Lin JZ, Li C, Farmer SR, Myocardin-related transcription factor A (MRTFA) regulates the fate of bone marrow mesenchymal stem cells and its absence in mice leads to osteopenia, Mol Metab. 2016; 5: 970-979.

5. Alexander KA, Chang MK, Maylin ER, Kohler T, Müller R, Wu AC, Van Rooijen N, Sweet MJ, Hume DA, Raggatt LJ, Pettit AR, Osteal macrophages promote in vivo intramembranous bone healing in a mouse tibial injury model, J Bone Miner Res. 2004; 26: 1517-1532.

6. Park JE, Barbul A, Understanding the role of immune regulation in wound healing, Am J Surg. 2004; 187: 11S-16S.

7. Vi L, Baht GS, Whetstone H, Ng A, Wei QX, Poon R, Mylvaganam S, Grynpas M, Alman BA, Macrophages promote osteoblastic differentiation in-vivo: implications in fracture repair and bone homeostasis,. J Bone Miner Res. 2015; 30: 1090-1102.

8. Schmidt-Bleek K, Petersen A, Dienelt A, Schwarz C, Duda GN, Initiation and early control of tissue regeneration - bone healing as a model system for tissue regeneration, Expert Opin Biol Ther. 2014; 14: 247-259.

9. Claes L, Recknagel $S$, Ignatius A, Fracture healing under healthy and inflammatory conditions, Nat Rev Rheumatol. 2012; 8: 133-143.

10. Parihar A, Eubank TD, Doseff Al, Monocytes and macrophages regulate immunity through dynamic networks of survival and cell death, J Innate Immun. 2010; 2: 204-215.

11. Chang B, Ma C, Liu X, Nanofibers regulate single bone marrow stem cell osteogenesis via FAK/RhoA/YAP1 pathway, ACS Appl Mater Interfaces. 2018; 10: 33022-33031.

12. Ma QL, Fang $L$, Jiang $N$, Zhang $L$, Wang $Y Y$, Zhang $M$, Chen $L H$, Bone mesenchymal stem cell secretion of SRANKL/OPG/M-CSF in response to macrophage-mediated inflammatory response influences osteogenesis on nanostructured Ti surfaces, Biomaterials. 2018; 154: 234-247.

13. Ren Z, Ma S, Jin L, Liu Z, Liu D, Zhang X, Cai Q, Yang X, Repairing a bone defect with a threedimensional cellular construct composed of a multi-layered cell sheet on electrospun mesh, Biofabrication. 2017; 9: 0

14. Pegtel DM, Gould SJ, Exosomes, Annu Rev Biochem. 2019; 88: 487-514.

15. Qiao L, Hu S, Liu S, Zhang H, Ma H, Huang K, Li Z, Su T, Vandergriff A, Tang J, Allen T, Dinh PU, Cores J, Yin Q, Li Y, Cheng K, microRNA-21-5p dysregulation in exosomes derived from heart failure patients impairs regenerative potential, J Clin Invest. 2019; 129: 2237-2250.

16. Zhang X, Sai B, Wang F, Wang L, Wang Y, Zheng L, Li G, Tang J, Xiang J, Hypoxic BMSC-derived exosomal miRNAs promote metastasis of lung cancer cells via STAT3-induced EMT, Mol Cancer. $2019 ; 18,40-54$.

17. Frank AC, Ebersberger S, Fink AF, Lampe S, Weigert A, Schmid T, Ebersberger I, Syed SN, Brune B, Apoptotic tumor cell-derived microRNA-375 uses CD36 to alter the tumor-associated macrophage phenotype, Nat Commun. 2019; 10: 1135-1147.

18. Cheng M, Yang J, Zhao X, Zhang E, Zeng Q, Yu Y, Yang L, Wu B, Yi G, Mao X, Hang K, Dong N, Xie M, Limdi NA, Prabhu SD, Zhang J, Qin G, Circulating myocardial microRNAs from infarcted hearts 
are carried in exosomes and mobilise bone marrow progenitor cells, Nat Commun. 2019; 10: 959974.

19. Xiong Y, Cao FQ, Hu LC, Yan CC, Chen L, Panayi AC, Sun Y, Zhou W, Zhang P, Wu QP, Xue H, Liu M, Liu Y, Liu J, Abududilibaier A, Mi B, Liu G, miRNA-26a-5p accelerates healing via downregulation of PTEN in fracture patients with traumatic brain injury, Mol Ther Nucleic Acids. 2019; 17: 223-234.

20. Nishimori S, O'Meara MJ, Castro CD, Noda H, Cetinbas M, da Silva Martins J, Ayturk U, Brooks DJ, Bruce M, Nagata M, Ono W, Janton CJ, Bouxsein ML, Foretz M, Berdeaux R, Sadreyev RI, Gardella TJ, Jüppner H, Kronenberg HM, Wein MN, Salt-inducible kinases dictate parathyroid hormone 1 receptor action in bone development and remodeling, J Clin 2019; 129: 51875203.

21. Schlundt C, El K, Serra A, Dienelt A, Wendler S, Schell H, van Rooijen N, Radbruch A, Lucius R, Hartmann S, Duda GN, Schmidt-Bleek K, Macrophages in bone fracture healing: Their essential role in endochondral ossification, B 2018; 106: 78-89.

22. Lewis JM, Vyas AD, Qiu Y, Messer KS, White R, Heller MJ, Integrated analysis of exosomal protein biomarkers on alternating current electrokinetic chips enables rapid detection of pancreatic cancer in patient blood, ACS Nan 2018; 12: 3311-3320.

23. Su T, Xiao Y, Xiao Y, Guo Q, Li C, Huang Y, Deng Q, Wen J, Zhou F, Luo XH, Bone marrow mesenchymal stem cells-derived exosomal miR-29b-3p regulates aging-associated insulin resistance, ACS Nano. 2019; 13: 2450-2462.

24. Thomou T, Mori MA, Dreyfuss JM, Konishi M, Sakaguchi M, Wolfrum C, Rao TN, Winnay JN, GarciaMartin R, Grinspoon SK, Gorden P, Kahn CR, Adipose-derived circulating miRNAs regulate gene expression in other tissues, Nature. 2017; 542: 450-455.

25. Yuan Z, Petree JR, Lee FE, Fan X, Salaita K, Guidot DM, Sadikot Macrophages exposed to HIV viral protein disrupt lung epithelial cell integrity and mitochondrial bioenergetics via exosomal microRNA shuttling, Cell Death Dis. 2019; 10, 580-594.

26. Ageta $\mathrm{H}$, Tsuchida $\mathrm{K}$, Post-translational modification and protein sorting to small extracellular vesicles including exosomes by ubiquitin and UBLs, Cell Mol Life Sci. 2019; 76: 4829-4848.

27. Yu Y, Newman H, Shen L, Sharma D, Hu G, Mirando AJ, Zhang H, Knudsen E, Zhang GF, Hilton MJ, Karner CM, Glutamine metabolism regulates proliferation and lineage allocation in skeletal stem cells, Cell Metab. 2019; 29: 966-978.

28. Ramanathan S, Shenoda BB, Lin ZC, Alexander GM, Huppert A, Sacan A, Ajit SK, Inflammation potentiates miR-939 expression and packaging into small extracellular vesicles, J Extracell Vesicles. 2019; 8: 1650595.

29. Sawada N, Jiang A, Takizawa F, Safdar A, Manika A, Tesmenitsky Y, Kang KT, Bischoff J, Kalwa H, Sartoretto JL, Endothelial PGC-1 a mediates vascular dysfunction in diabetes, Cell Metab. 2014; 19: 246-258.

30. Lombardi MS, Gilliéron C, Berkelaar M, Gabay C, Salt-inducible kinases (SIK) inhibition reduces RANKL-induced osteoclastogenesis,. Plos One. 2017; 12: e0185426. 
31. Darling NJ, Toth R, Arthur JS, Clark K, Inhibition of SIK2 and SIK3 during differentiation enhances the anti-inflammatory phenotype of macrophages, Biochem J. 2017; 474: 521-537.

32. Lin C, Yu S, Jin R, Xiao Y, Pan M, Pei F, Zhu X, Huang H, Zhang Z, Chen S, Circulating miR-338 cluster activities on osteoblast differentiation: potential diagnostic and therapeutic targets for postmenopausal osteoporosis, Theranostics. 2019; 9: 3780-3797.

33. Li CJ, Xiao Y, Yang M, Su T, Sun X, Guo Q, Huang Y, Luo XH, Long noncoding RNA Bmncr regulates mesenchymal stem cell fate during skeletal aging, J Clin Invest. 2018; 128: 5251-5266.

34. Li Y, Yang F, Gao M, Gong R, Jin M, Liu T, Sun T, Fu Y, Huang Q, Zhang W, miR-149-3p regulates the switch between adipogenic and osteogenic differentiation of BMSCs by targeting FTO, Mol Ther Nucleic Acids. 2019; 17: 590-600.

35. Ricarte FR, Le Henaff C, Kolupaeva VG, Gardella TJ, Partridge NC, Parathyroid hormone(1-34) and its analogs differentially modulate osteoblastic Rankl expression via PKA/SIK2/SIK3 and PP1/PP2ACRTC3 signaling, J Biol Chem. 2018; 293: 20200-20213.

36. Kim MK, Kwon JO, Song MK, Kim B, Kim H, Lee ZH, Koo SH, Kim HH, Salt-inducible kinase 1 regulates bone anabolism via the CRTC1-CREB-Id1 axis, Cell Death Dis. 2019; 10: 826-837.

\section{Tables}

Table 1. miRNAs and mRNA primer sequence. 


\begin{tabular}{|c|c|}
\hline microRNAs or gene name & Primer sequence ( $5^{\prime}$ to $\left.3^{\prime}\right)$ \\
\hline mmu - miR - 5106 - Forward & CTCAACTGGTGTCGTGGAGTCGGCAATTCAGTTGAGTCTGCCAA \\
\hline mmu - miR - 5106 - Reverse & TCTGTAGCTCAGTTGGCAGACTC \\
\hline mmu - U6-Forward & GCTTCGGCAGCACATATACTAAAAT \\
\hline mmu - U6-Reverse & CGCTTCACGAATTTGCGTGTCAT \\
\hline mmu - SIK2 - Forward & AGAAGCAGUCUCAGCUGCAAGCAU \\
\hline mmu - SIK2 - Reverse & UAUGCUUGCAGCUGAGACUGCUUC \\
\hline mmu - SIK3 - Forward & CCACAUGCUGGUGUUAGAUCCAAA \\
\hline mmu - SIK3 - Reverse & AUUUGGAUCUAACACCAGCAUGUG \\
\hline mmu - Col 1a1 - Forward & CTGACTGGAAGAGCGGAGAG \\
\hline mmu - Col 1a1 - Reverse & CGGCTGAGTAGGGAACACAC \\
\hline mmu - ALP - Forward & TGACTACCACTCGGGTGAACC \\
\hline mmu - ALP - Reverse & TGATATGCGATGTCCTTGCAG \\
\hline mmu - OCN - Forward & TTCTGCTCACTCTGCTGACCC \\
\hline mmu - OCN - Reverse & CTGATAGCTCGTCACAAGCAGG \\
\hline mmu - Runx2 - Forward & CGCCACCACTCACTACCACAC \\
\hline mmu - Runx2 - Reverse & TGGATTTAATAGCGTGCTGCC \\
\hline mmu - GAPDH - Forward & AGAGTGTTTCCTCGTCCCG \\
\hline mmu - GAPDH - Reverse & CCGTTGAATTTGCCGTGA \\
\hline
\end{tabular}

\section{Figures}


A
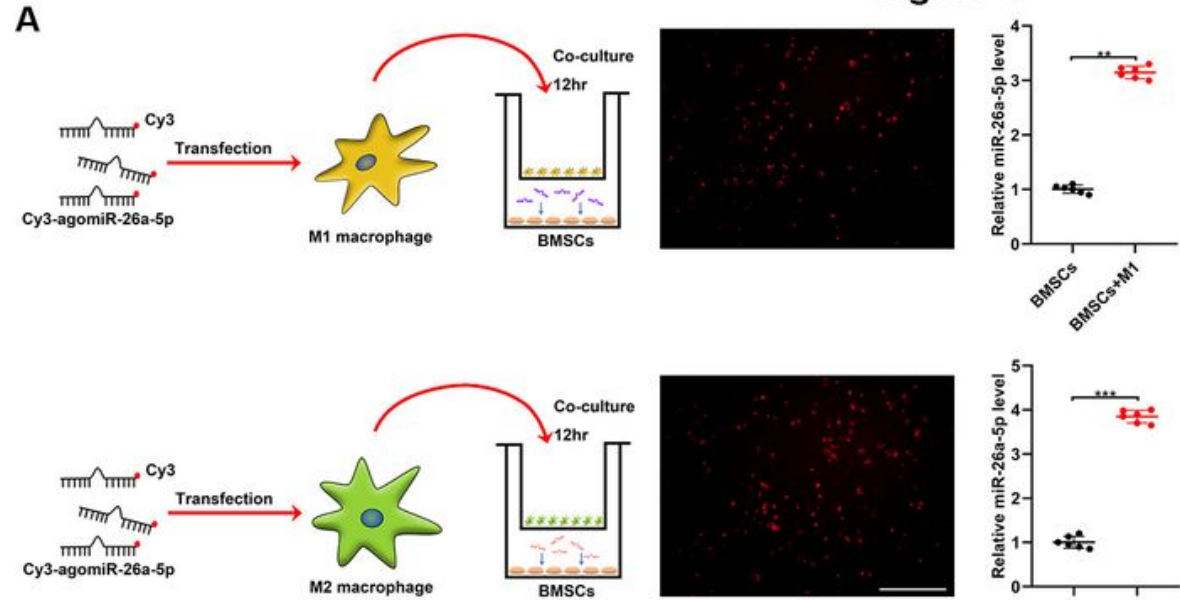

C

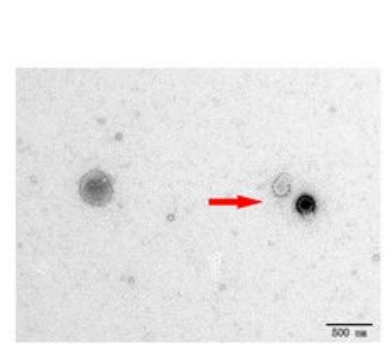

M1D-Exos

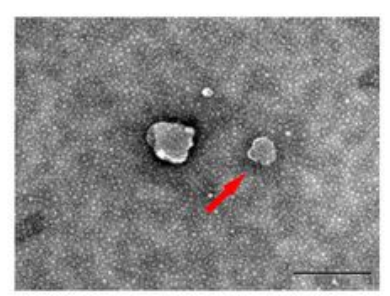

M2D-Exos

E

BMSCs+ M1D-Exos
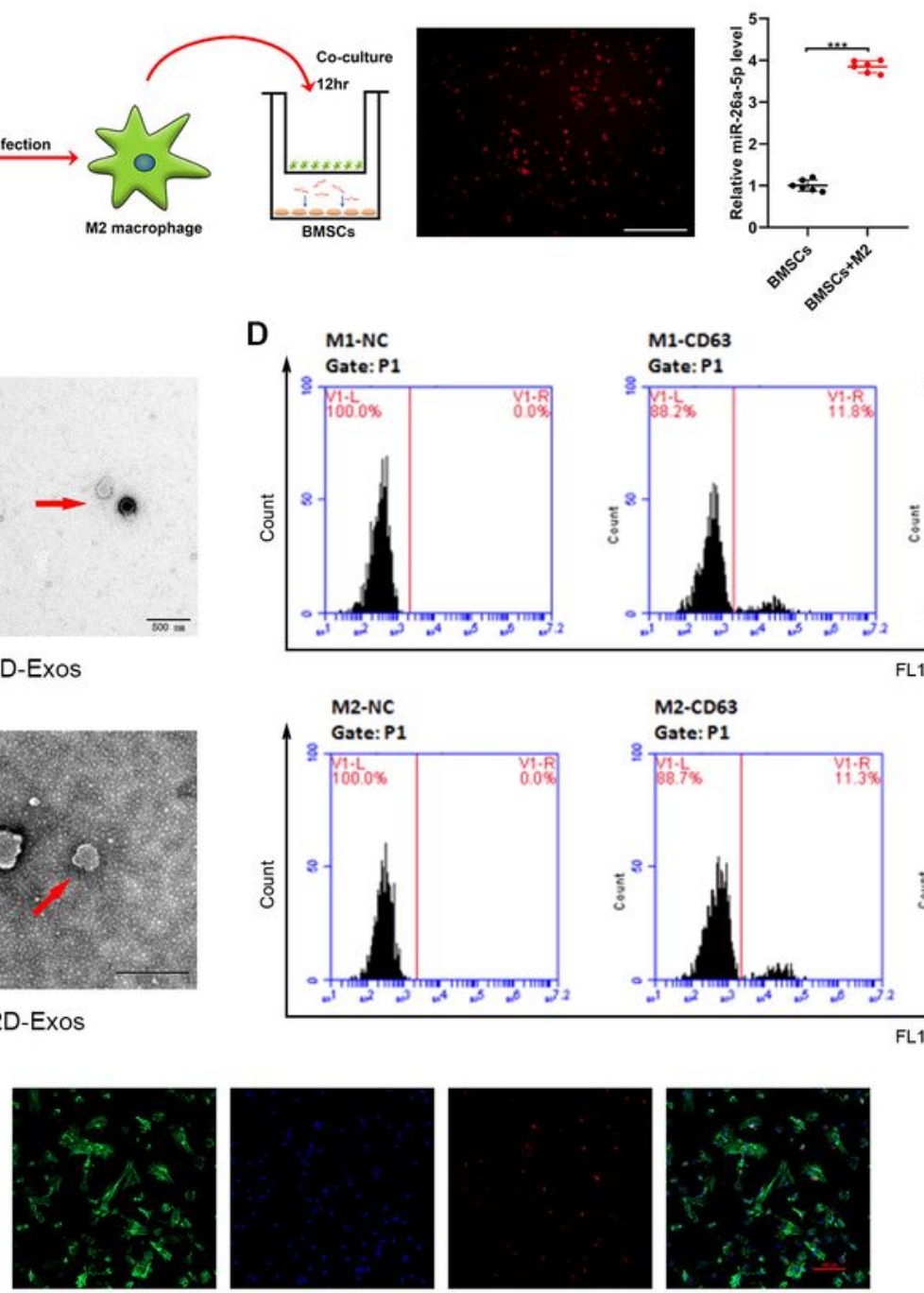

B
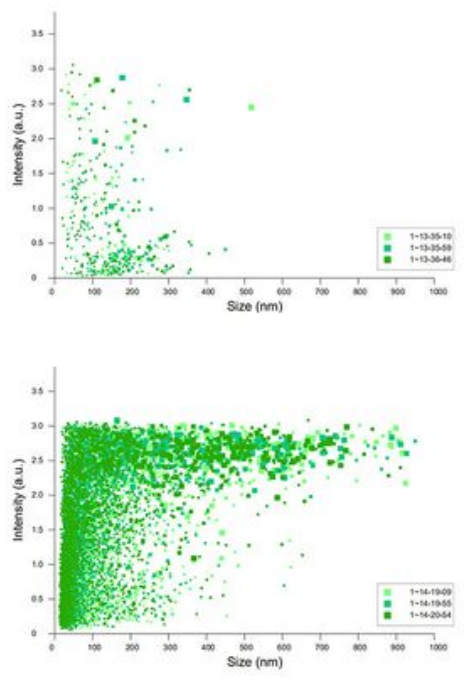

M1-NC

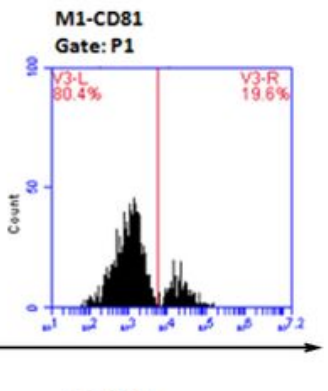

M2-NC
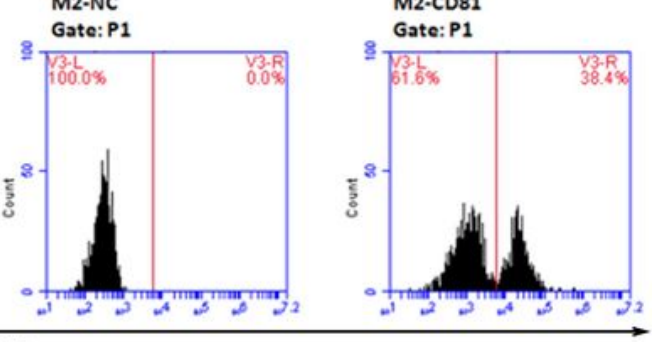

FL1-A

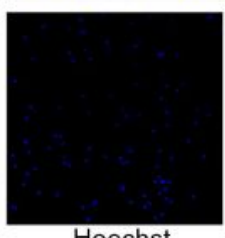

Hoechst

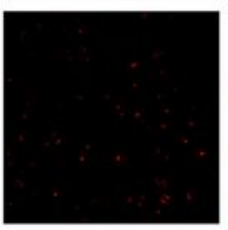

PKH-26

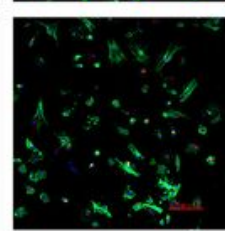

Merge

\section{Figure 1}

Macrophages secrete exosomal miRNAs. (A) M1 and M2 macrophages transfected with a Cy3-labeled miR-26a-5p mimic were co-cultured with BMSCs in a transwell (membrane pore $=0.4 \mathrm{~nm}$ ) plate; $(B)$ Particle size of the vesicles secreted from M1 and M2 macrophages were measured by NanoSight analysis; (C) Represent picture of the ultrastructure of the two kind of exosomes observed by TEM. Scar bar = $200 \mathrm{~nm}$; (D) The protein levels of CD 63 and CD 81 in the two kind exosomes; (E) The two kind of 
exosomes were marked with red flurescence dye PKH26 and co-cultured with BMSCs, red flurescence represents exosomes in BMSCs, scar bar $=50 \mu \mathrm{m}$. Data are means \pm SD of triplicate experiments. ${ }^{*} \mathrm{p}<$ $0.05, * * p<0.01, * * * p<0.001$.

A

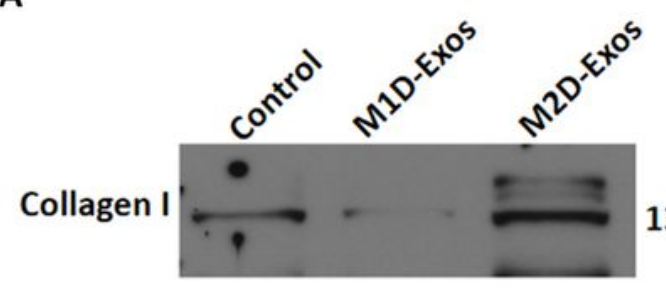

Figure 2
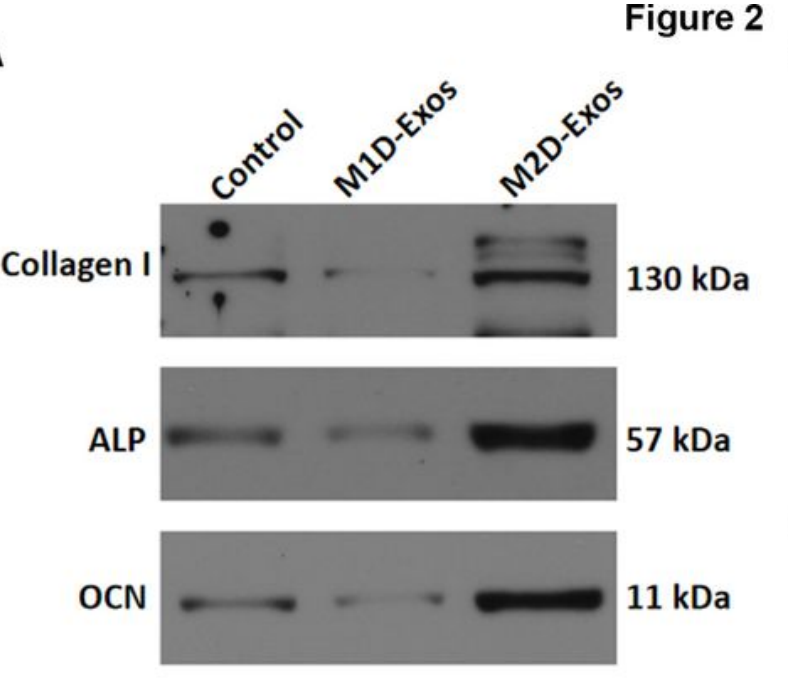

11 kDa
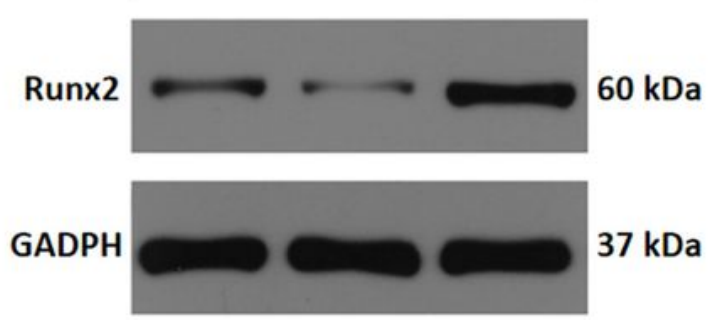

$37 \mathrm{kDa}$
B

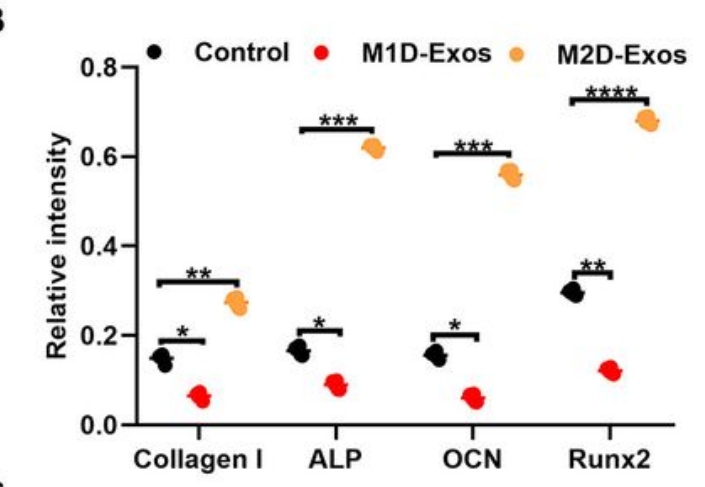

C

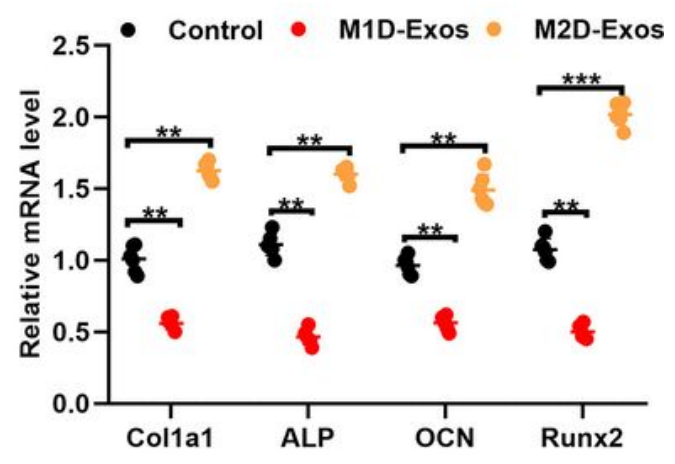

D
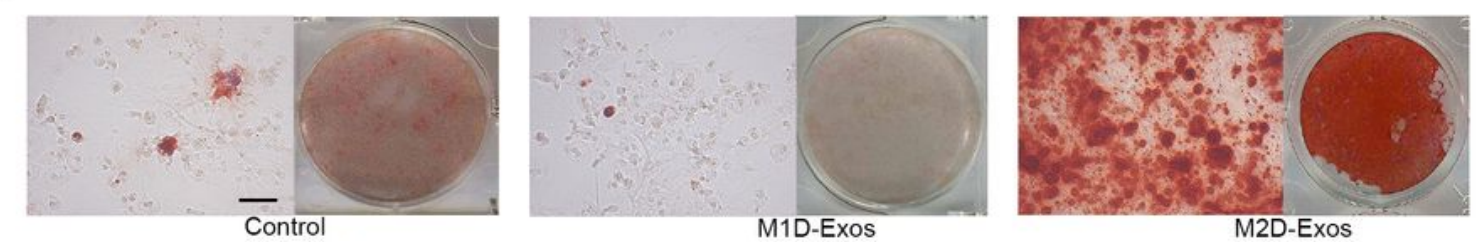

E

SIK 2 and SIK 3 expressions
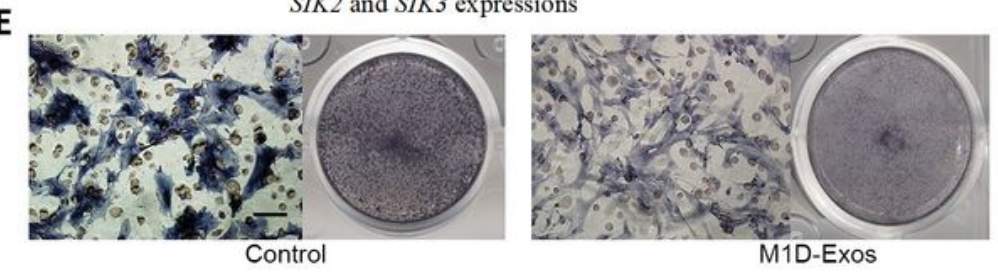

M1D-Exos

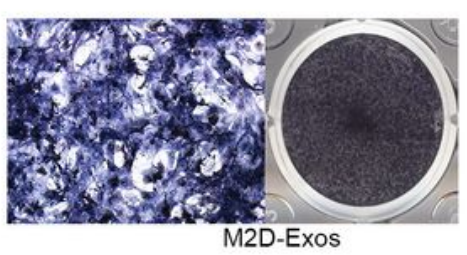

$\mathbf{F}$

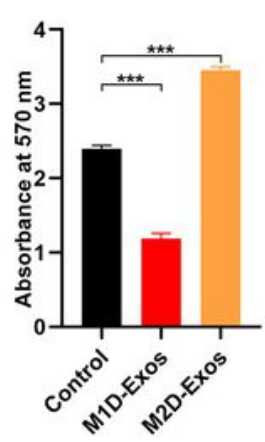

G

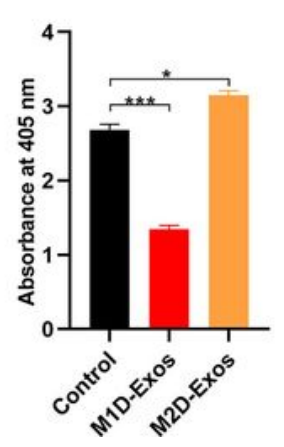

Figure 2

M2D-Exos induce osteoblast activity and matrix mineralization. (A) Osteogenic genes were upregulated in M2D-Exos-treated BMSCs measured by western blotting analysis; (B) The relative intensity of western 
blotting analysis; (C) Overexpression of the four osteognic genes can be detected in M2D-Exos groups measured by qRT-PCR analysis; (D) Alizarin red-mediated calcium staining in BMSCs following treated by PBS (control group), M1D-Exos, and M2D-Exos for 21 days. Scale bar = 10mm; (E) ALP staining in BMSCs following treated by PBS (control group), M1D-Exos, and M2D-Exos for 14 days. Scale bar $=10 \mathrm{~mm}$; (F-G) The statistical data of Alizarin red-mediated calcium staining and ALP staining. Data are means \pm SD of triplicate experiments. ${ }^{*} p<0.05,{ }^{*} p<0.01,{ }^{* *} p<0.001$.

A

Control
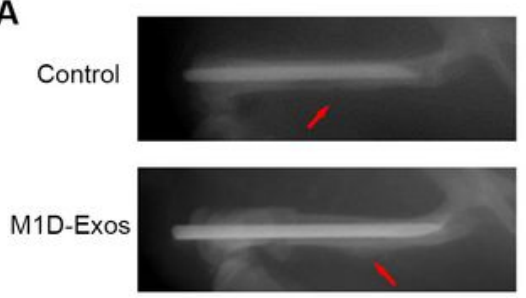

M2D-Exos

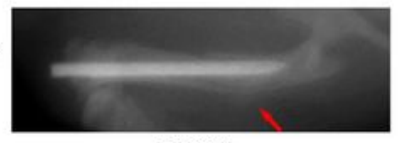

DAY 3
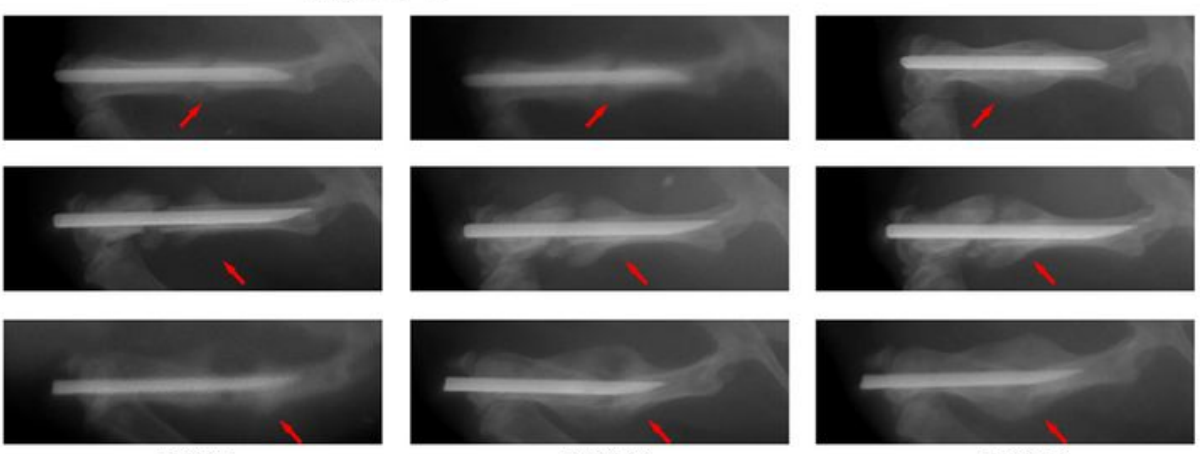

DAY 7

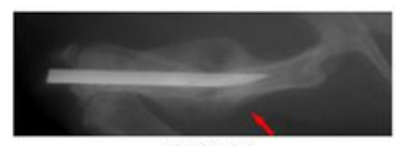

DAY 14
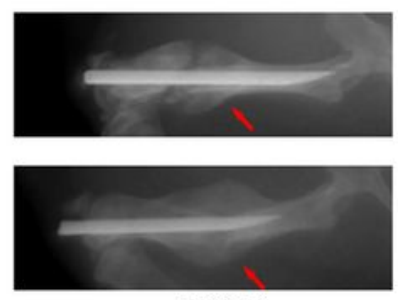

DAY 21

B
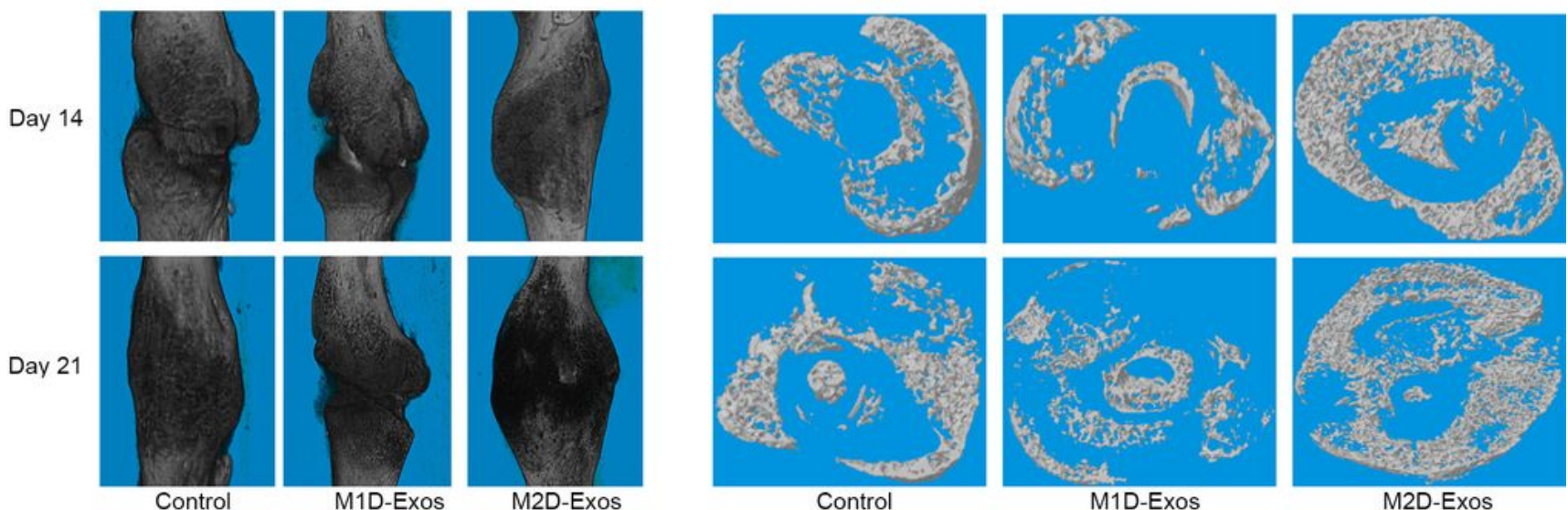

C

D
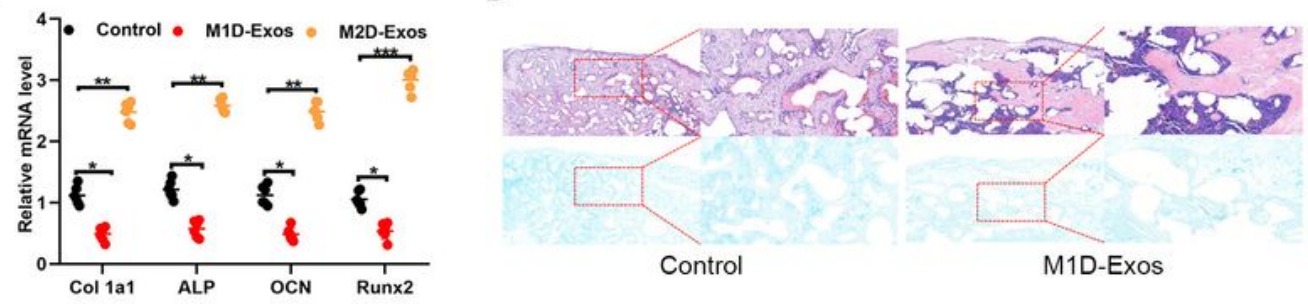

Control

M1D-Exos

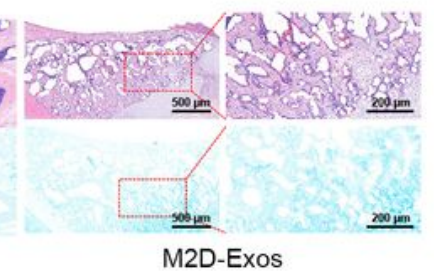

E

F
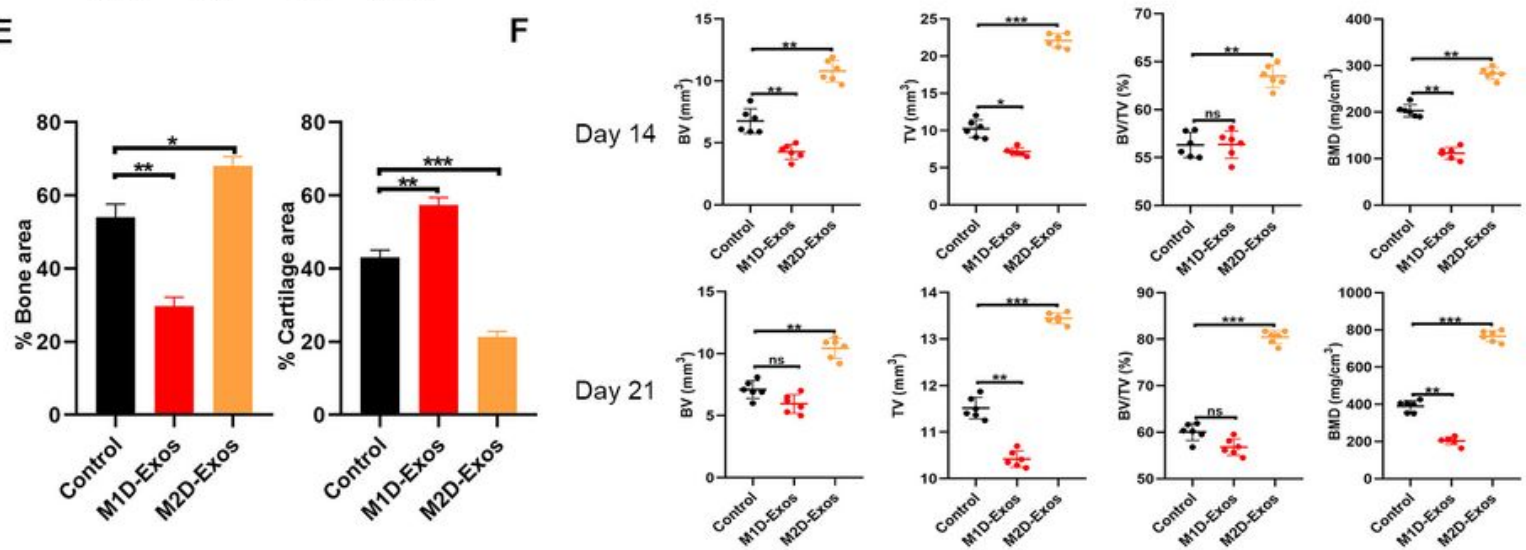

Figure 3 
M2D-Exos accelerates fracture healing in vivo. (A) X-rays comparison of fracture healing between control, M1D-Exos, and M2D-Exos groups on day 3, 7, 14 and 21 post-injury; (B) micro-CT three dimensional construction and cross sections of the fracture site among the three groups on day 14 and 21 ; (C) Osteogenic expression levels were measured by qRT-PCR analysis, $n=6$ mice/group; (D) The H\&E/Alcianblue staining images on day 21 post-operation. (E) The statistical results of H\&E/Alcian-blue staining; (F) BV and TV of the callus, BV/TV, and BMD datas on days 14 and 21 post-operation were assessed via micro-CT. $n=6$ mice/group. Data are means \pm SD of triplicate experiments. ${ }^{\star} p<0.05,{ }^{\star \star} p<0.01,{ }^{\star \star \star} p<$ 0.001 . 
A

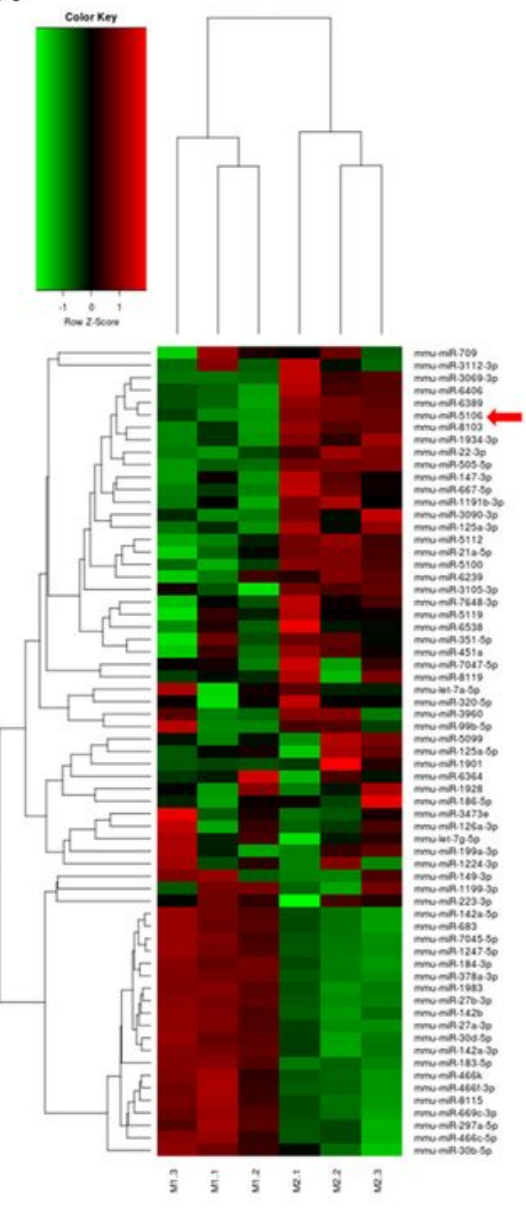

D

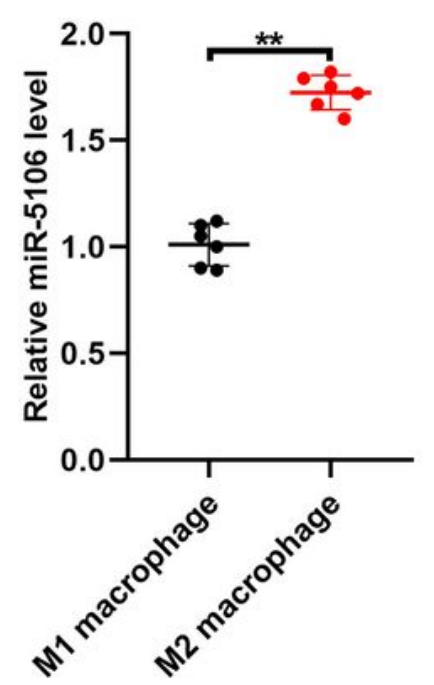

B

Figure 4

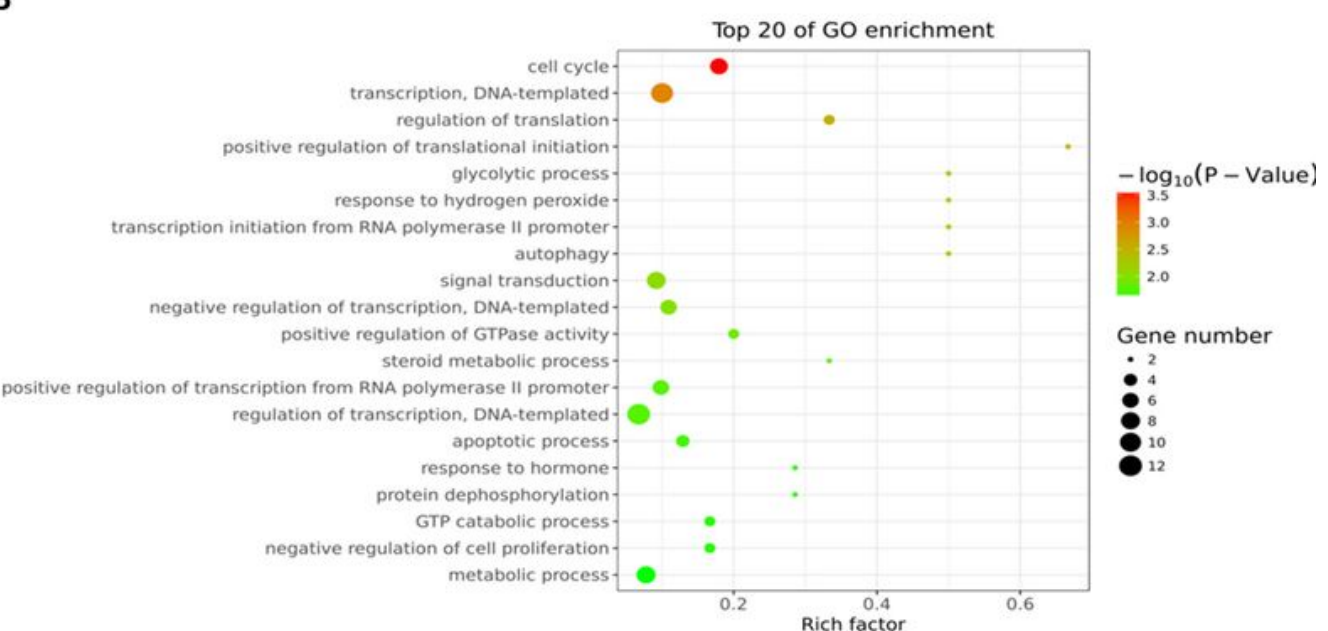

C

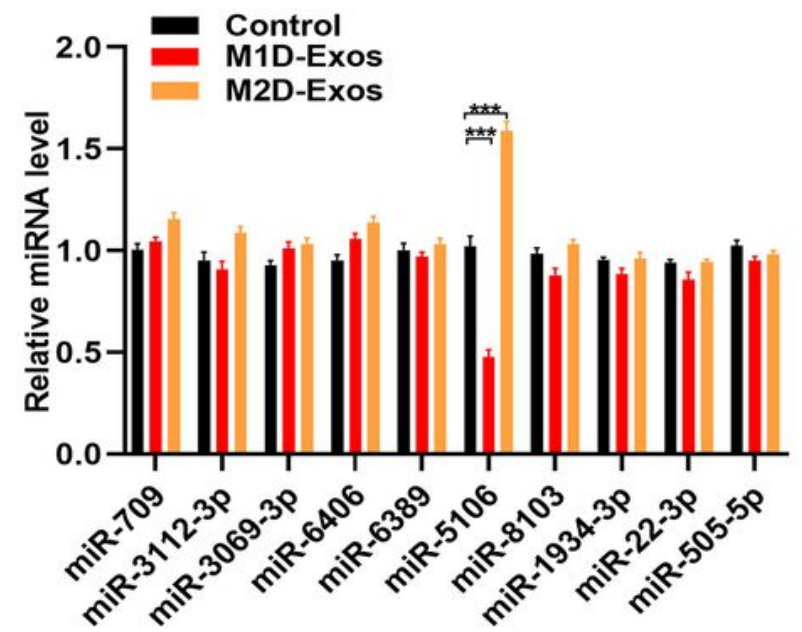

E

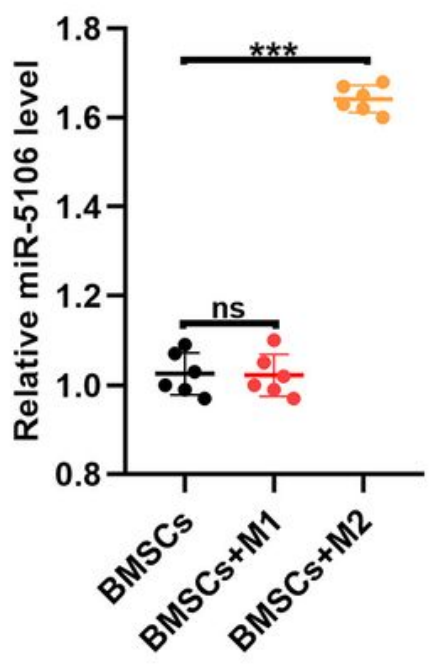

$F$

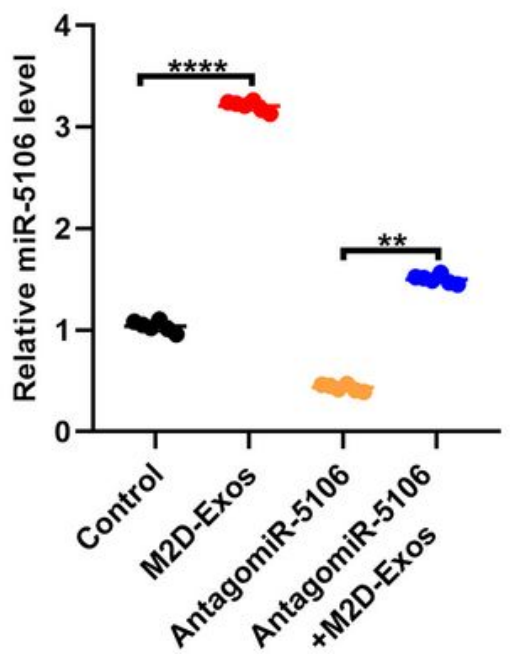

Figure 4

Identification of differentially expressed miRNAs between M1D-Exos and M2D-Exos. (A) Heat map identified the differently expressed miRNAs (fold change $>5$ or $<-5$, Benjamini-Hochberg-corrected $p$ ); (B) The GO (gene ontology) enrichment of the differently expressed miRNAs; (C) Expression of the top ten differentially expressed miRNAs between M1D-Exos and M2D-Exos; (D) The miR-5106 level between M1 and M2 macrophages; (E) The miR-5106 level among groups following co-cultured BMSCs with M1 or M2 
macrophages; (F) The miR-5106 level among the groups with different treatments. Data are means \pm SD. ${ }^{*} \mathrm{p}<0.05,{ }^{* *} \mathrm{p}<0.01,{ }^{* * *} \mathrm{p}<0.001$.

A

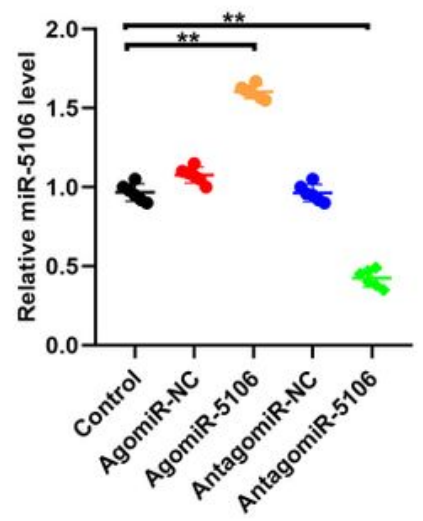

B

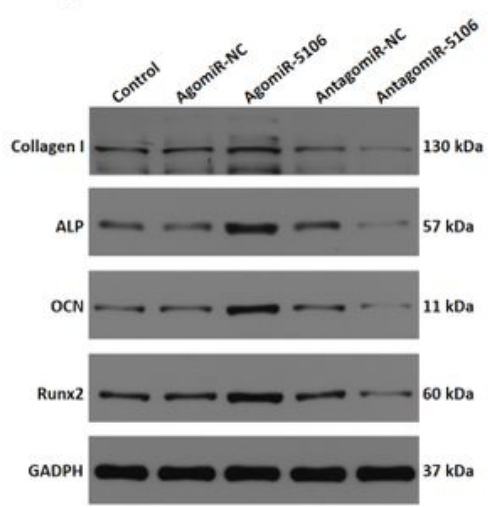

$E$

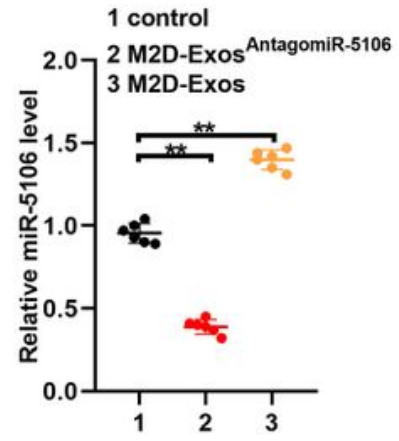

Figure $5 \quad \mathrm{C}$

$\mathrm{H}$

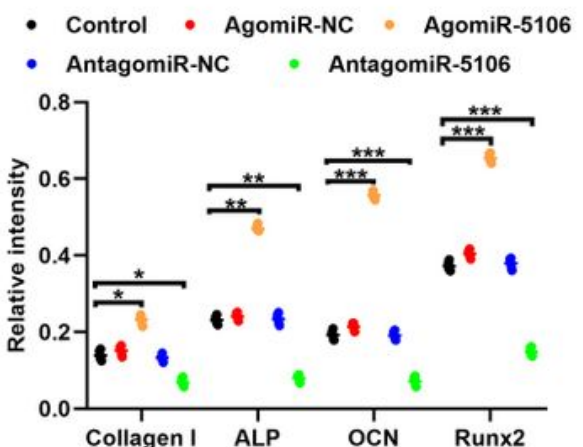

$\mathbf{F}$

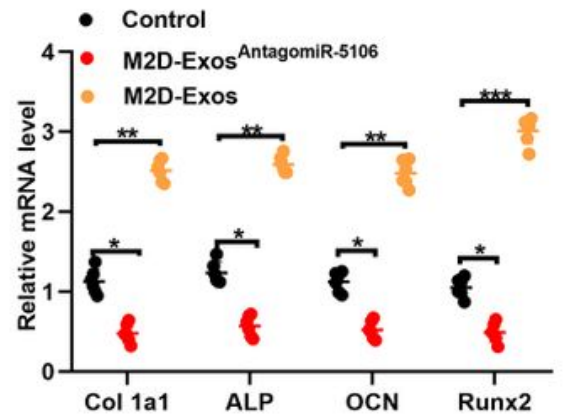

G

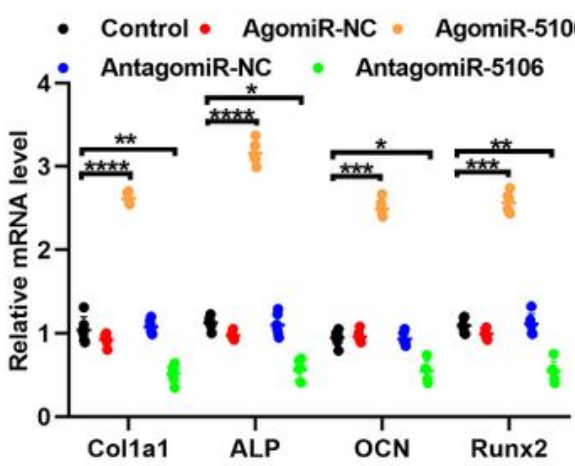

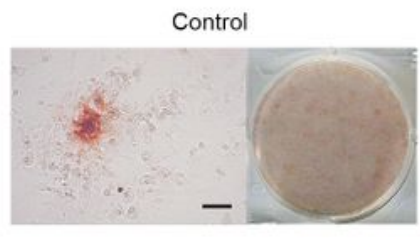

AgomiR-5106

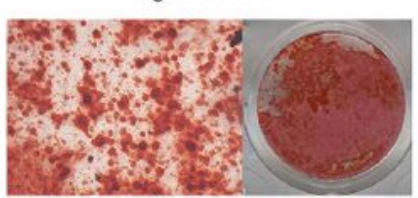

AntagomiR-5106

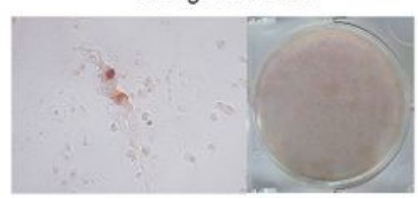

AgomiR-NC

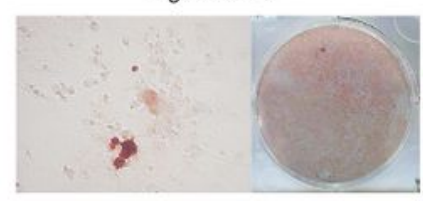

AntagomiR-NC
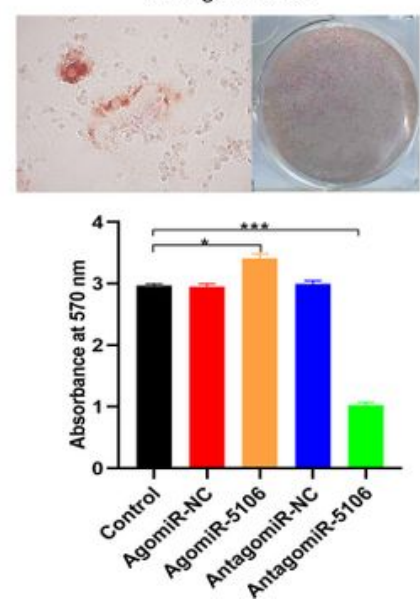

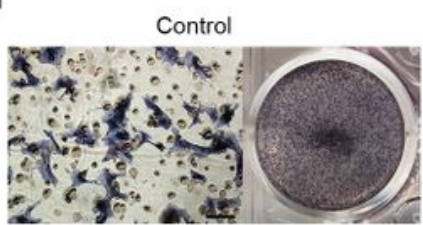

AgomiR-5106
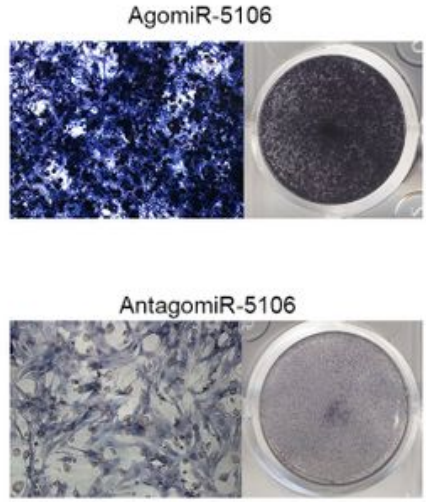

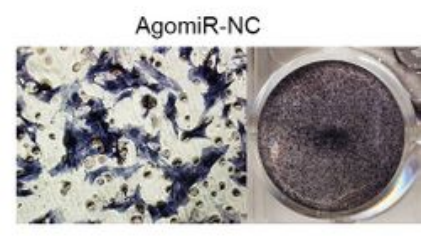

AntagomiR-NC
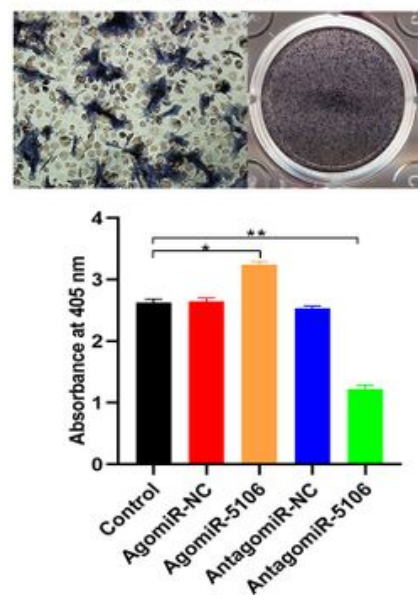

Figure 5

M2D-Exos induce osteoblastic differentiation of BMSCs in vitro. (A) miRNA-5106 was upregulated in agomiR-5106 treated BMSCs following transfection using lipofecamine control, 200 $\mu \mathrm{M}$ agomiR-5106, agomiR-NC, antagomiR-5106 or antagomiR-NC for 48 hours; (B) Western blotting analysis of Collagen I, 
ALP, OCN and Runx2 protein levels in BMSCs treated using lipofecamine control, agomiR-NC, agomiR5106, antagomiR-NC or antagomiR-5106 for 48 hours; (C) The relative intensity of the western blotting analysis; (D) qRT-PCR analysis was used to assess expression of osteoblast differentiation genes including Col1a1, ALP, OCN , and Runx2 in transfected BMSCs; (E) M2D-Exos were treated with miR-5106 inhibitor (antagomiR-5106) to investigate the effect of M2D-Exos with miR-5106-silence on the osteoblastic differentiation of BMSCs. The results showed that antagomiR-5106 can significantly decrease the miR-5106 expression in M2D-Exos; (F) Decreased osteogenic genes expression can be detected in M2D-ExosantagomiR-5106 group compared with PBS and M2D-Exos groups; (G) Alizarin redmediated calcium staining in BMSCs following differently transfeced for 21 days, Scale bar $=10 \mathrm{~mm} ;(H)$ ALP staining in BMSCs following different treatments for 14 days. Scale bar $=10 \mathrm{~mm}$. Data are means \pm SD. ${ }^{\star} p<0.05,{ }^{\star \star} p<0.01,{ }^{\star * \star} p<0.001$. 
A

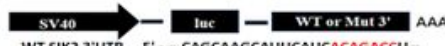

WT SIK2 3'UTR $5^{\circ}$ - CAGCAAGCAUUCAUCACAGACCU -

miR-5106 3'-- AGACGGUUGACUCGAUGUCUGGA-

MUT SIK2 3'UTR ' S'- CAGCAAGCAUUCAUCUGUCUGGU -

WT SIK3 3'UTR S' -- UUCCACAGCCUGUCU--CAGACCAG-

mir-5106 3'-- AGACGGUUGACUCGaUGUCUGGA

MUT SIK3 J'UTR S' -- UUCCACAGCCUGUCU-UGUCUGGG -

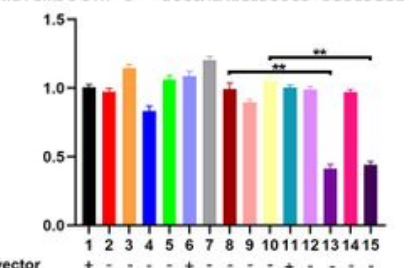

Lue vector

LUC-SIK2.WT $\ldots+\ldots \ldots+\ldots \ldots+\cdots$

Luc-SIK3-Mut . . . . . . . . +

LuC-SIK3-WT $\ldots \ldots+\ldots+\ldots+$

AgomiR-NC

AgomiR-s106

F

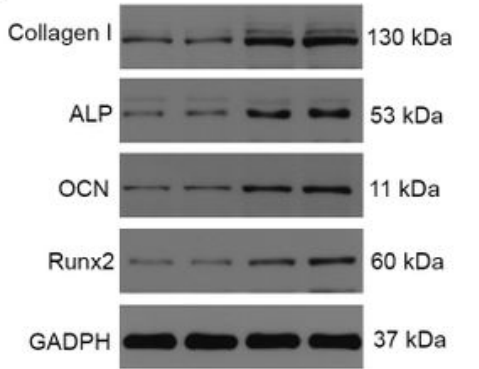

I

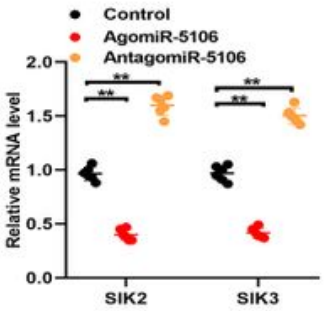

B

Figure 6

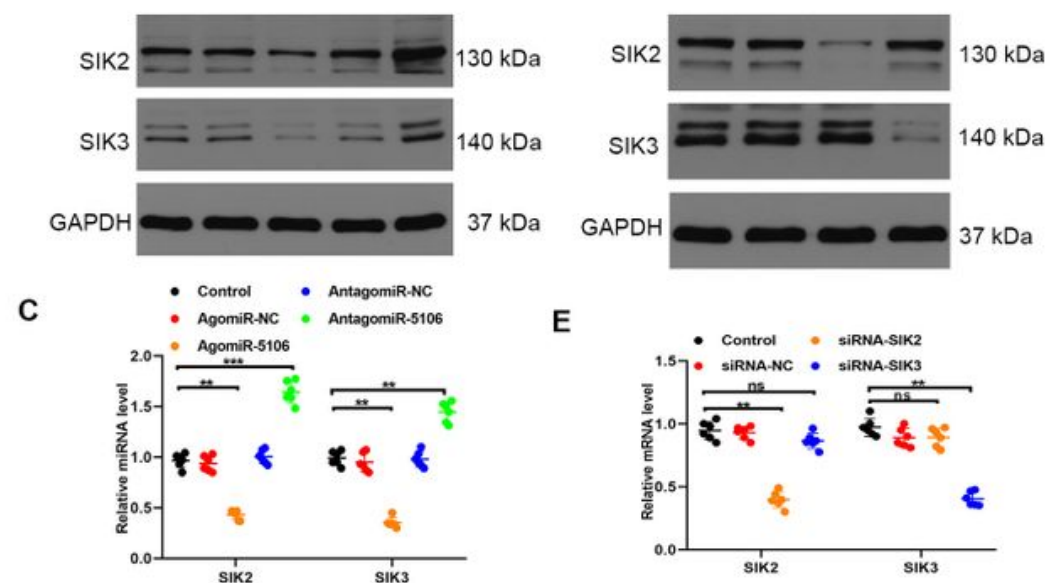

G

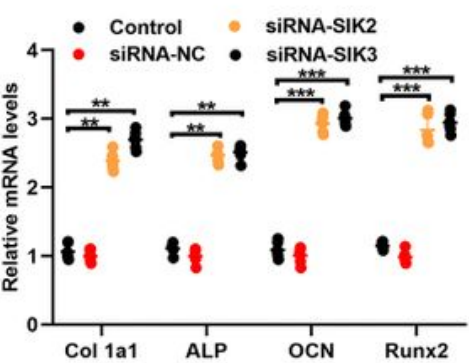

H

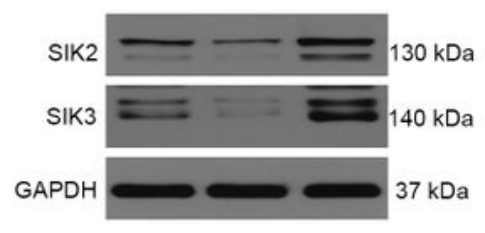

L

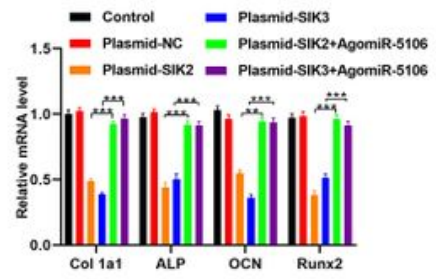

SIRNA-SIK3

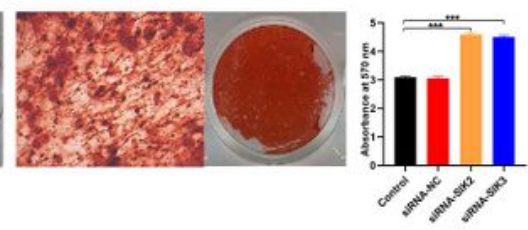

SIRNA-SIK3

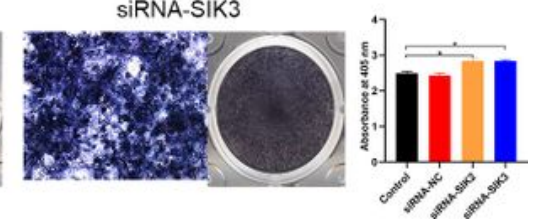

Figure 6

miRNA-5106 targets SIK2 and SIK3 to induce osteoblastic activity in vitro. (A) Luciferase reporter constructs containing either a WT SIK2 and SIK3 3' UTR (WT 3' UTR) or this same region after sitedirected mutagenesis (3' UTR-Mut). Luc, luciferase. Endogenous miRNA-5106 effects in BMSCs on WT SIK2 and SIK3 3' UTR (luc-UTR), the SIK2 and SIK3 3' UTR mutant (luc-UTR-Mut), as assessed following agomiR-NC or agomiR-5106 treatment; (B) The levels of mRNA SIK2 and SIK3 were western blotting 
analysis in agomiR-5106 treated BMSCs following transfection using lipofecamine control, $200 \mu \mathrm{M}$ agomiR-NC, agomiR-5106, antagomiR-NC or antagomiR-5106 for 48hours; (C) The expression of SIK2 and SIK3 was measured by qRT-PCR analysis; (D-E) Western blotting and qRT-PCR analysis were used following control, siRNA-NC, siRNA-SIK2, and siRNA-SIK3 transfection to assess SIK2 and SIK3 expression, with a scrambled siRNA utilized as a negative control (siRNA-NC); (F-G) The levels of osteogenic genes were measured by western qRT-PCR and blotting analysis; $(\mathrm{H}-\mathrm{I})$ SIK2 and SIK3 expressions in mice callus decreased significantly on day 14 after receiving the injections of agomiR5106 compared with PBS and antagomiR-5106 groups; (J-K) SIK2 and SIK3 expressions were measures by qRT-PCR analysis following different treatments; (L) Osteogenic related genes level was assessed by qRT-PCR analysis; (M) Alizarin red-mediated calcium staining and (N) ALP staining in BMSCs following differently transfected, Scale bar $=10 \mathrm{~mm}$. Data are means \pm SD. ${ }^{*} p<0.05,{ }^{\star \star} p<0.01,{ }^{\star \star \star} p<0.001$. 
A

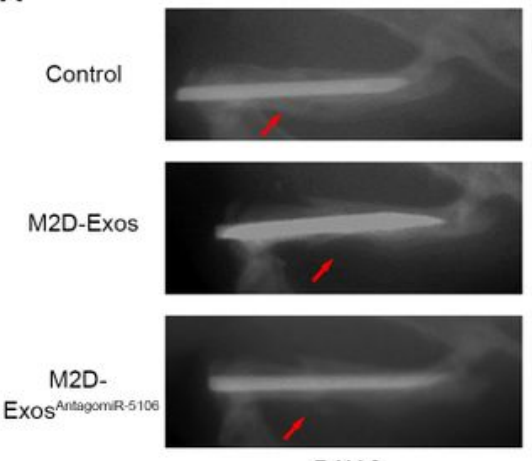

DAY 3

B
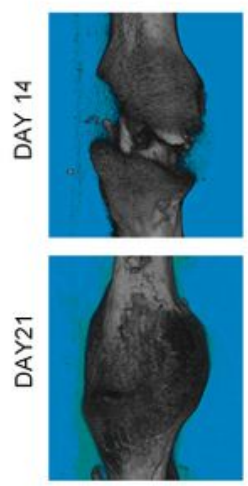

Control

C

1. Control
2. M2D-Exos

3. M2D-Exos ${ }^{\text {Antagomir-5106 }}$

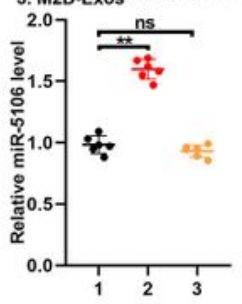

E

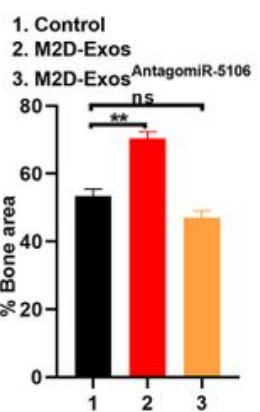

M2D-Exos

D
Figure 7
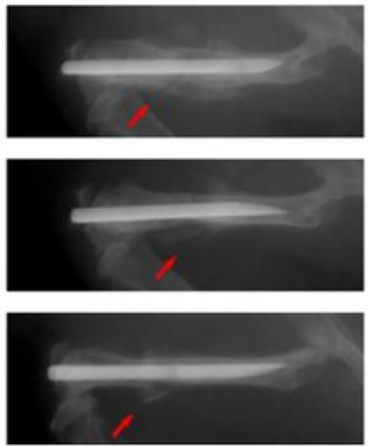

DAY 7
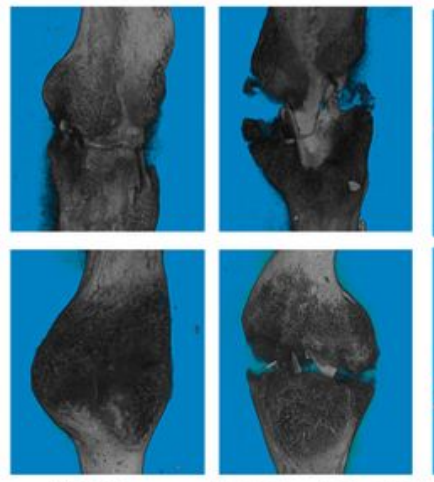

M2D-EXos AntagomR 5106
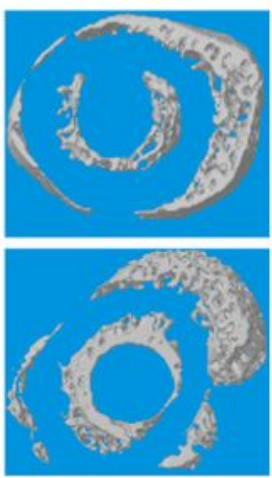

Control

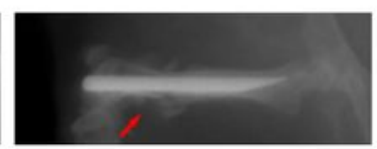

DAY 14
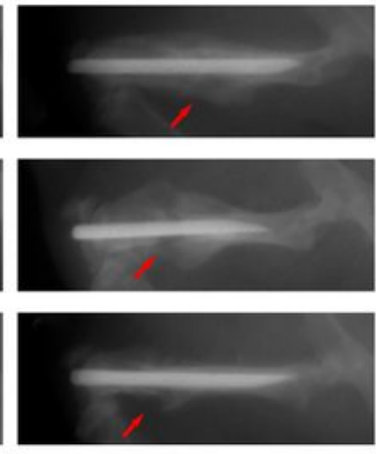

DAY21
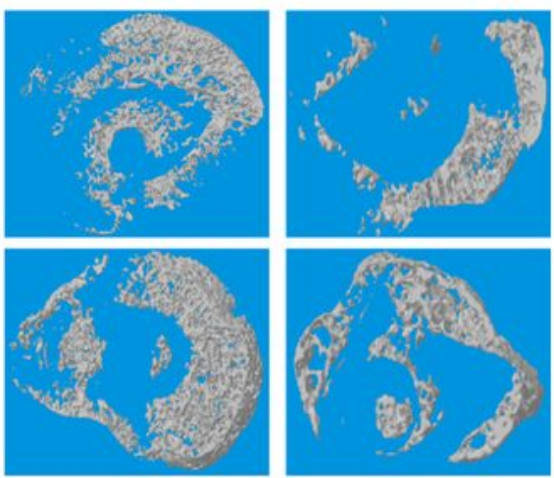

M2D-Exos

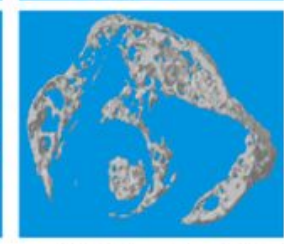

M2D-Exos Antapomir.5100
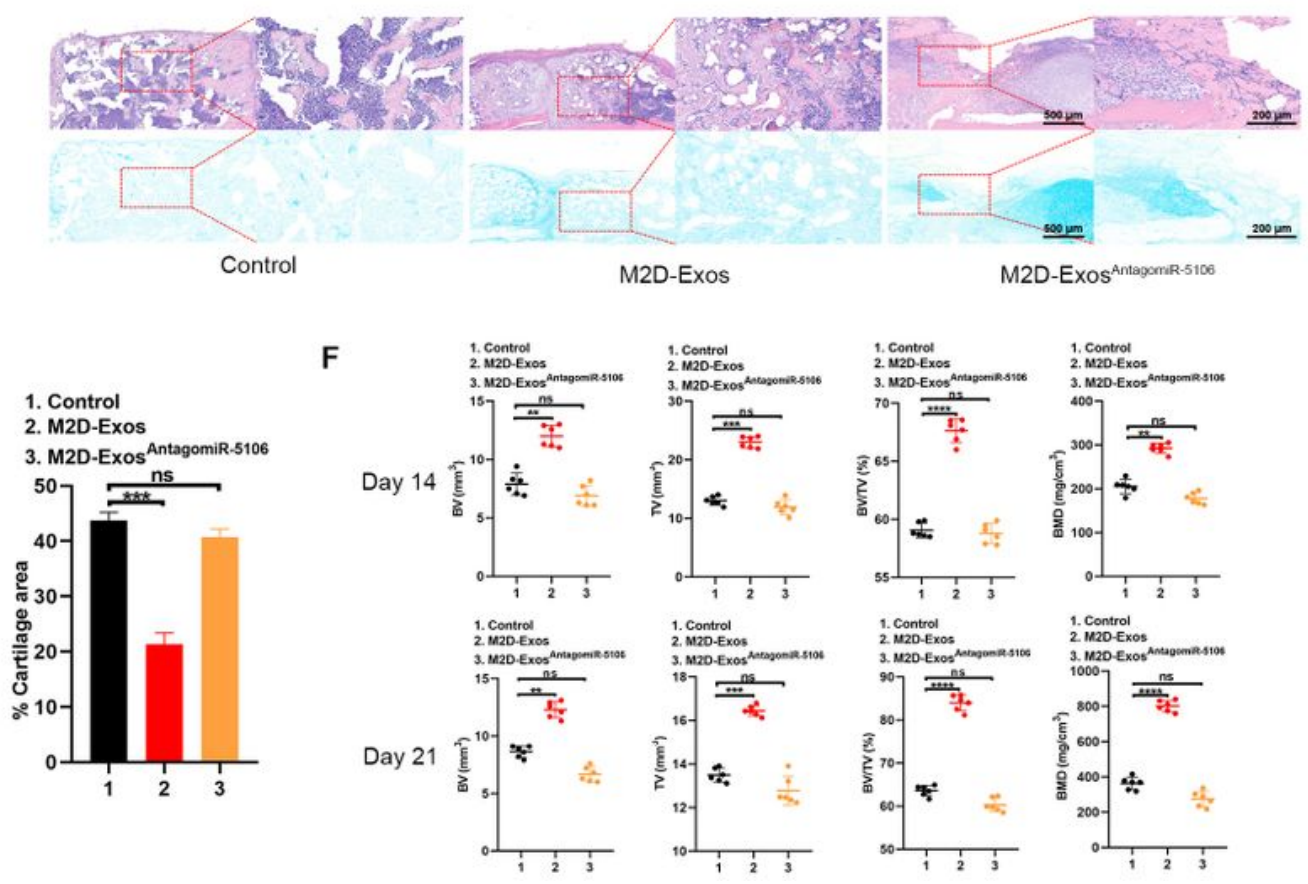

Figure 7

miR-5106 accelerates fracture healing in vivo. (A) X-rays comparison of fracture healing between control, M2D-Exos, and M2D-ExosantagomiR-5106 groups on day 3, 7, 14 and 21 post-injury; (B) micro-CT three dimensional construction and cross sections of the fracture site among the three groups on day 14 and 21 ; (C) miR-5106 level in callus was measured by qRT-PCR analysis, $n=6$ mice/group; (D) The H\&E/Alcian-blue staining images on day 21 post-operation. (E) The statistical results of H\&E/Alcian-blue 
staining; (F) BV and TV of the callus, BV/TV, and BMD datas on days 14 and 21 post-operation were assessed via micro-CT. $n=6$ mice/group. Data are means \pm SD of triplicate experiments. ${ }^{\star} p<0.05,{ }^{\star} \mathrm{p}<$ $0.01, * \star * p<0.001$.

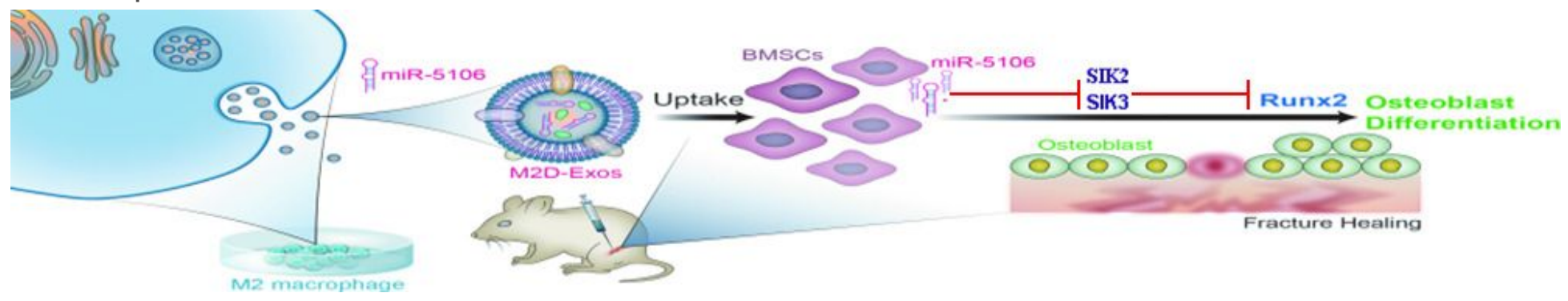

Figure 8

Schematic diagram showing proposed mechanisms by which exosomal miR-5106 derived from M2 macrophages induce the BMSCs towards osteoblastic differentiation. Enriched level of miR-5106 in MEDExos enhances the inhibitory effect on SIK2 and SIK3. Decreased SIK2 and SIK3 expression induces BMSCs differentiation and thereby accelerates bone remodeling.

\section{Supplementary Files}

This is a list of supplementary files associated with this preprint. Click to download.

- Supplementaryinformation.pdf 